\title{
Nuclear obscuration in LINERs
}

\section{Clues from Spitzer/IRS spectra on the Compton thickness and the existence of the dusty torus ${ }^{\star}$}

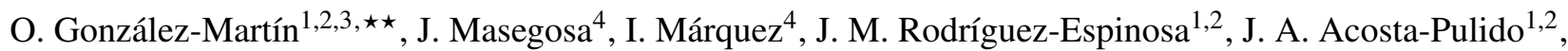 \\ C. Ramos Almeida ${ }^{1,2}$, D. Dultzin ${ }^{5}$, L. Hernández-García ${ }^{4}$, D. Ruschel-Dutra ${ }^{1,2,6}$, and A. Alonso-Herrero ${ }^{7,8}$ \\ 1 Instituto de Astrofísica de Canarias (IAC), C/vía Láctea, s/n, 38205 La Laguna, Spain \\ e-mail: o.gonzalez@crya.unam.mx \\ 2 Departamento de Astrofísica, Universidad de La Laguna (ULL), 38205 La Laguna, Spain \\ 3 Centro de Radioastronomía y Astrofísica (CRyA-UNAM), 3-72 (Xangari), 8701 Morelia, Mexico \\ 4 Instituto de Astrofísica de Andalucía, CSIC, Glorieta de la Astronomía s/n, 18008 Granada, Spain \\ 5 Instituto de Astronomía, Universidad Nacional Autónoma de México, Apartado Postal 70-264, 04510 México DF, Mexico \\ ${ }^{6}$ Departamento de Astronomia, Universidade Federal do Rio Grande do Sul, 9500 Bento Goncalves, 91501-970, Porto Alegre, Brazil \\ 7 Instituto de Física de Cantabria, CSIC-UC, 39005 Santander, Spain \\ 8 Department of Physics and Astronomy, University of Texas at San Antonio, San Antonio, TX 78249, USA
}

Received 31 October 2014 / Accepted 15 January 2015

\section{ABSTRACT}

Context. Most of the optically classified low-ionisation, narrow emission-line regions (LINERs) nuclei host an active galactic nucleus (AGN). However, how they fit into the unified model (UM) of AGN is still an open question.

Aims. The aims of this work are to study at mid-infrared (mid-IR) (1) the Compton-thick nature of LINERs (i.e. hydrogen column densities of $N_{\mathrm{H}}>1.5 \times 10^{24} \mathrm{~cm}^{-2}$ ) and (2) the disappearance of the dusty torus in LINERs predicted from theoretical arguments. Methods. We have compiled all the available low spectral-resolution, mid-IR spectra of LINERs from the InfraRed Spectrograph (IRS) onboard Spitzer. The sample contains 40 LINERs. We have complemented the LINER sample with Spitzer/IRS spectra of PG QSOs, Type-1 Seyferts (S1s), Type-2 Seyferts (S2s), and StarBurst (SB) nuclei. We studied the AGN compared to the starburst content in our sample using different indicators: the equivalent width of the polycyclic aromatic hydrocarbon at $6.2 \mu \mathrm{m}$, the strength of the silicate feature at $9.7 \mu \mathrm{m}$, and the steepness of the mid-IR spectra. We classified the spectra as SB-dominated and AGN-dominated, according to these diagnostics and compared the average mid-IR spectra of the various classes. Moreover, we studied the correlation between the $12 \mu \mathrm{m}$ luminosity, $v L_{v}(12 \mu \mathrm{m})$, and the $2-10 \mathrm{keV}$ energy band X-ray luminosity, $L_{\mathrm{X}}(2-10 \mathrm{keV})$.

Results. In 25 out of the 40 LINERs (i.e. 62.5\%), the mid-IR spectra are not SB-dominated, similar to the comparison S2 sample (67.7\%). The average spectra of both SB-dominated LINERs and S2s are very similar to the average spectrum of the SB class. The average spectrum of AGN-dominated LINERs is different from the average spectra of the other optical classes, showing a rather flat spectrum at 6-28 $\mu \mathrm{m}$. We find that the average spectrum of AGN-dominated LINERs with X-ray luminosities $L_{\mathrm{X}}(2-10 \mathrm{keV})>$ $10^{41} \mathrm{erg} / \mathrm{s}$ is similar to the average mid-IR spectrum of AGN-dominated S2s. However, faint LINERs (i.e. $\left.L_{\mathrm{X}}(2-10 \mathrm{keV})<10^{41} \mathrm{erg} / \mathrm{s}\right)$ show flat spectra different from any of the other optical classes. The correlation between $v L_{\nu}(12 \mu \mathrm{m})$ and $L_{\mathrm{X}}(2-10 \mathrm{keV})$ for AGN nicely extends towards low luminosities only if SB-dominated LINERs are excluded and if the $2-10 \mathrm{keV}$ band X-ray luminosity is corrected in Compton-thick LINER candidates.

Conclusions. We find that LINERs proposed as Compton-thick candidates at X-ray wavelengths may be confirmed according to the $\mathrm{X}$-ray to mid-IR luminosity relation. We show evidence that the dusty-torus disappear when their bolometric luminosity is below $L_{\text {bol }} \simeq 10^{42} \mathrm{erg} / \mathrm{s}$. We suggest that the dominant emission at mid-IR of faint LINERs might be a combination of an elliptical galaxy host (characterised by the lack of gas), a starburst, a jet, and/or ADAF emission. Alternatively, the mid-IR emission of some of these faint LINERs could be a combination of elliptical galaxy plus carbon-rich planetary nebulae. To reconcile the Compton-thick nature of a large number of LINERs without dusty-torus signatures, we suggest that the material producing the Compton-thick X-ray obscuration is free of dust.

Key words. galaxies: active - galaxies: nuclei - infrared: galaxies

\section{Introduction}

The emission in active galactic nuclei (AGNs) is powered by accretion onto a supermassive black hole (SMBH). AGNs are traditionally divided into two main classes based on the presence (Type-1) or not (Type-2) of broad permitted lines (FWHM > $2000 \mathrm{~km} \mathrm{~s}^{-1}$ ) in the optical spectrum. The so-called unification model (UM) proposes that both types of AGNs are essentially the same objects viewed at different angles (Antonucci

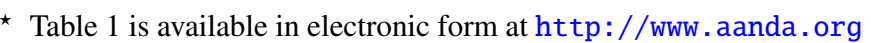
$\star \star$ Juan de la Cierva Fellow and Tenure track at CRyA.
}

1993; Urry \& Padovani 1995). An optically thick dusty torus surrounding the central source would then be responsible for blocking the region where these broad emission lines are produced (the broad line region, BLR) in Type-2 Seyferts. The torus must not be spherically symmetric, in order to obscure the BLR, while at the same time allowing the emission coming from region producing the permitted narrow lines (known as narrowline region, NLR) to reach us from the same line of sight (LOS).

Low-ionisation nuclear emission-line regions (LINERs), first classified by Heckman (1980), are the dominant population of AGN in the local Universe (Ho et al. 1997). However, they remain as one of the most captivating subsets of nuclear classes 
because their main physical mechanism is still unknown. The nature of LINERs was initially sustained in their optical spectrum, which can be reproduced with a variety of different physical processes (e.g. photoionisation from hot stars, non-thermal photoionisation, shocks, post-main sequence stars, or AGN, Dopita \& Sutherland 1995; Heckman 1980; Ferland \& Netzer 1983; Veilleux \& Osterbrock 1987; Stasińska et al. 2008; Singh et al. 2013). In fact, many authors have suggested that LINERs are a heterogeneous class (Satyapal et al. 2005; Dudik et al. 2005, 2009). Indeed the emission mechanism dominating their optical spectrum is still under debate. Singh et al. (2013) show that a single AGN cannot explain the radial profile of the surface brightness $\mathrm{H} \alpha$ emission line, so a contribution of extended emission post-main sequence stars is needed at optical frequencies. That this contribution can be seen in LINERs might be the intrinsic weakness of the AGNs, which would outshine these signatures in more powerful AGNs. In line with that, GonzálezMartín et al. (2014) show that the host-galaxy contributes a large amount in most of the LINERs even in X-rays. Thus, the analysis of LINERs could be key to studying the interplay between the AGN and the host galaxy.

Nowadays we know that around $75-90 \%$ of LINERs show evidence of AGNs using multi-wavelength information (González-Martín et al. 2006, 2009a, 2014; Dudik et al. 2009; Younes et al. 2011; Asmus et al. 2011; Mason et al. 2012) ${ }^{1}$. This is also confirmed from X-ray and UV variability studies (Maoz et al. 2005; Hernández-García et al. 2013, 2014). However, what makes LINERs different from other AGNs? How do they fit into the UM of AGNs? Some results suggest that they might constitute a class of AGN with a different accretion mode (e.g. Younes et al. 2011; Nemmen et al. 2014), while some other authors have argued that large obscuration is responsible for their differences (e.g. Dudik et al. 2009; González-Martín et al. 2009b). González-Martín et al. (2009a) found that the hydrogen column density, $N_{\mathrm{H}}$, in LINERs range from the galactic value up to $N_{\mathrm{H}} \simeq 10^{24} \mathrm{~cm}^{-2}$. This is fully consistent with the $N_{\mathrm{H}}$ values reported for Seyfert galaxies (e.g. Panessa et al. 2006; Bianchi et al. 2012; Marinucci et al. 2012). However, using the ratio between the luminosity of the [OIII] $\lambda 5007 \AA$ emission line and the intrinsic hard $(2-10 \mathrm{keV}) \mathrm{X}$-ray luminosity, $\mathrm{L}([\mathrm{OIII}]) / L_{\mathrm{X}}(2-10 \mathrm{keV})$, as a tracer of Compton-thickness (i.e. $N_{\mathrm{H}}>1.5 \times 10^{24} \mathrm{~cm}^{-2}$ ), González-Martín et al. (2009b) found that up to $53 \%$ of the LINERs in their sample are Compton-thick candidates. This percentage is two times higher than reported for Type-2 Seyferts (Maiolino et al. 1998; Bassani et al. 1999; Panessa et al. 2006; Cappi et al. 2006). Dudik et al. (2009) studied the emission lines in 67 high-resolution Spitzer/IRS spectra of LINERs and find that the central power source in a high percentage of LINERs is highly obscured at optical frequencies, consistent with the X-ray results.

Obscuring dust hampers the studies of the optical to soft $\mathrm{X}$-ray emission coming from both young hot-stars and the accretion disk. However, emission at mid-infrared (mid-IR) wavelengths does not suffer such high extinction. Furthermore, the dust that absorbs the shorter wavelength emission reradiates in the mid-IR and correspondingly produces a substantial fraction of the bolometric flux of the object. Dissecting the detailed midIR spectra of AGN can reveal the properties of the dust in the nuclear region (e.g. Mendoza-Castrejón et al. 2015). Subarcsecond resolution studies claim a tight correlation between rest frame

\footnotetext{
1 Most of these studies select their sources using archival X-rays observations so they might be biased by the complex selective effect that this introduces into the sample.
}

luminosities $v L_{v}(12 \mu \mathrm{m})$ and $L_{\mathrm{X}}(2-10 \mathrm{keV})$ for Type-1 and Type-2 Seyferts (Horst et al. 2009; Gandhi et al. 2009; Asmus et al. 2011; Masegosa et al. 2013; González-Martín et al. 2013). Imaging data at mid-IR wavelengths have shown that some Compton-thick LINER candidates might fall off this relation, with $L_{12 \mu \mathrm{m}}$ higher than expected for their $L_{\mathrm{X}}$ (Mason et al. 2012; Masegosa et al. 2013). The confirmation of such a trend for a large sample might confirm the Compton-thick nature of a large portion of LINERs.

From the theoretical point of view, Elitzur \& Shlosman (2006) show that the torus might disappear when the bolometric luminosity decreases below $L_{\text {bol }} \sim 10^{42} \mathrm{erg} / \mathrm{s}$ because the accretion onto the SMBH can no longer sustain the required cloud outflow rate. Thus, the low bolometric luminosity of LINERs makes them important for probing this theoretical prediction. Maoz et al. (2005) show that the fraction of variable Type- 1 and Type-2 LINERs at UV is the same. This favours the lack of a dusty torus obscuring the central AGN in Type-2 LINERs since the central source is what is responsible for such a variability pattern. Müller-Sánchez et al. (2013) also found some evidence in favor of such disappearance using observations of molecular gas in three prototypical low-luminosity AGN (LLAGN). The mid-IR emission shows clear signatures of the dusty torus. In fact, clumpy torus models (Nenkova et al. 2008) have succeeded in explaining the mid-IR emission of Seyfert galaxies (e.g. Ramos Almeida et al. 2009, 2011; Alonso-Herrero et al. 2011; Hönig et al. 2010). Thus, the mid-IR spectra of LINERs can give important clues to the existence of the dusty torus for low bolometric luminosities. However, other contributors, such as jet emission (e.g. NGC 1052, Fernández-Ontiveros et al. 2012) or optically thin dust (e.g. NGC 3998, Mason et al. 2013), can also contribute to the mid-IR emission.

The purpose of this paper is to study (1) the Compton-thick nature of LINERs and (2) the plausible disappearance of the torus. We present the mid-IR Spitzer/IRS spectra of 40 LINERs. We compare them with mid-IR Spitzer/IRS spectra of Seyferts, PG QSOs, and starbursts (SBs) (see Sect. 2). The data reduction and measurements are described in Sect. 3. The relatively low spatial resolution of Spitzer/IRS spectra means that these spectra are contaminated from the host galaxy emission, which is particularly relevant for LLAGN as LINERs. Section 4 describes a method that is able to select those mid-IR spectra with a negligible SB contribution. Section 5 studies the average spectrum of LINERs and compares them with that of Seyferts, PG QSOs, and SBs. Section 6 shows the analysis of the correlation between $L_{\mathrm{X}}(2-10 \mathrm{keV})$ and $L_{12} \mu \mathrm{m}$ for LINERs. In Sect. 7 we discuss the implications of the main results. The conclusions of this paper are summarised in Sect. 8.

\section{Sample}

Our initial sample of LINERs comes from the catalog of LINERs observed at X-rays published by González-Martín et al. (2009a). This guarantees that all the LINERs have $L_{X}(2-10 \mathrm{keV})$ measurements, which is crucial for our purposes. However, we must be aware that this sample does not constitute a complete sample. For the present analysis we have used two databases to obtain the Spitzer data for an additional sample of LINERs. The first one is the Cornell atlas of Spitzer/IRS spectra (CASSIS ${ }^{2}$ ). CASSIS provides low-resolution spectra $(R \sim 60-127$ over $5.2 \mu \mathrm{m}$ to $38 \mu \mathrm{m}$ ) with the IRS instruments in the stare mode (Lebouteiller et al. 2011). The second database is the Spitzer infrared nearby galaxy survey (SINGS Kennicutt et al. 2003).

2 http://cassis.astro. cornell.edu/atlas/ 
SINGS is a Legacy programme of imaging and spectroscopic data for 75 nearby galaxies. As part of the Legacy programme, all the one-dimensional nuclear spectra have been archived in the infrared science archive (IRSA ${ }^{3}$ ). This provides uniform 5-30 $\mu \mathrm{m}$ spectra in the spectral mapping mode. Most galaxies in the SINGS sample have also been observed with Chandra, and their main X-ray properties are described by Grier et al. (2011).

The CASSIS atlas contains 27 LINERs published by González-Martín et al. (2009a). We have also added NGC 3079, which was not analysed by González-Martín et al. (2009a), but it was included as a Compton-thick LINER by Goulding et al. (2012). Grier et al. (2011) included 20 LINERs (8 included in González-Martín et al. 2009a).

The final sample of LINER in this paper contains 40 Spitzer/IRS spectra. Among them, eight have been optically classified as Type-1.9 LINERs (LINER1) and 32 as Type-2 LINERs (LINER2) by Ho et al. (1997). Three of them are known Compton-thick, 21 are Compton-thin, and 16 were classified as Compton-thick candidates by González-Martín et al. (2009b).

Dudik et al. (2009) report mid-IR spectra of 67 LINERs (13 objects in common with our sample). However, they used the high-resolution modes of Spitzer/IRS because their work was focused on the fine structure mid-IR emission lines. Thus, their results are not directly comparable to ours. Sturm et al. (2006) report a mid-IR study of 33 LINERs. They selected their sample on the basis of IR luminosity, while our sample is conformed by objects with measured X-ray luminosities. As a consequence, only NGC 4486 is in common with our sample.

\subsection{Comparison samples}

To study the possible contribution of star-formation or AGN emission, we have selected SBs, Seyferts, and PGQSOs to be able to compare their mid-IR spectra to those of LINERs. The sample is not complete in any sense, but it allows us to have a representative set of objects for each category to compare LINERs with them:

- Seyferts. All the Type-1 and Type-2 sources are included in Shi et al. (2006), in the Compton-thick sample described by Goulding et al. (2012), and in the SINGS sample. In total it contains 42 Seyferts. Among the 31 are Type-2 Seyferts (S2, including 19 Compton-thick and 12 Compton-thin) and 11 Type-1 Seyferts (S1) ${ }^{4}$.

- Palomar Green QSOs (PG QSOs). This sample includes all the PG QSOs in the sample defined by Veilleux et al. (2009) with Spitzer/IRS spectra in CASSIS and redshifts $z<0.2^{5}$. This PG QSO sample includes 26 sources.

- Starbursts. This sample is taken from Ranalli et al. (2003), Brandl et al. (2006), and Grier et al. (2011). The SB sample contains 21 sources. Among them NGC 3367 was classified as a Seyfert by Véron-Cetty \& Véron (2006), although it was classified as a SB by Ho et al. (1997).

For all the classes, we have only included spectra observed with both the short-low (SL) and long-low (LL) modules to guarantee the full Spitzer/IRS coverage (at least $\sim 5-30 \mu \mathrm{m}$ ). Moreover, ultra-luminous infrared galaxies (ULIRGs) have been excluded

\footnotetext{
3 http://irsa.ipac.caltech.edu

4 The S1 sample contains objects classified as Type 1, Type 1.2, Type 1.5 , Type 1.8 , and Type 1.9 Seyferts. The $\mathrm{S} 2$ sample only includes purely Type-2 Seyferts.

5 The redshift limit has been chosen to be able to obtain rest-frame $30 \mu \mathrm{m}$ luminosities, as required for our analysis.
}

from the analysis because they might have a controversial source of emission at mid-IR (Imanishi et al. 2007; Alonso-Herrero et al. 2013). All together these samples comprise 89 sources (129 nuclei including LINERs).

\section{Data processing and analysis}

CASSIS and SINGS provide flux- and wavelength-calibrated spectra. However, the observations using data from both the SL and LL spectral modules suffer from mismatches due to telescope-pointing inaccuracies or due to different spatial resolutions of the IRS orders. This is not corrected in the final products given by CASSIS and SINGS. We therefore scaled each spectrum to the immediate prior (in wavelength range) to overcome such effects. Thus, our flux level is scaled to the level of the shortest wavelengths, which is the order with the highest spatial resolution. This guarantees that the flux level is scaled to the best spatial resolution that Spitzer can provide. Moreover, the spectra are shifted to rest frame according to the redshift of the objects.

For each object we have measured the $12 \mu \mathrm{m}$ and $30 \mu \mathrm{m}$ luminosities using the Spitzer/IRS spectra. Errors were estimated assuming $15 \%$ flux-calibration uncertainties, which fully dominate other source of errors (e.g. González-Martín et al. 2013; Ramos Almeida et al. 2011). We also measured the fluxes and equivalent width (EW) of the polycyclic aromatic hydrocarbon (PAH) features at 6.2 and $11.3 \mu \mathrm{m}$. The EW of the PAH features were measured by integrating the emission over the continuum in a wavelength range of 5.9-6.5 $\mu \mathrm{m}$ and $11.0-11.6 \mu \mathrm{m}$ for the 6.2 and $11.3 \mu \mathrm{m}$ PAH emission features, respectively. The continuum was estimated through a linear fit to the 5.5-5.9 $\mu \mathrm{m}(10.7-11.0 \mu \mathrm{m})$ and 6.5-6.7 $\mu \mathrm{m}(11.6-11.9 \mu \mathrm{m})$ for the $6.2 \mu \mathrm{m}(11.3 \mu \mathrm{m})$ PAH feature (see e.g. González-Martín et al. 2013). We also computed the strength of the silicate emission/absorption feature at $9.7 \mu \mathrm{m}$ through the apparent depth at $9.7 \mu \mathrm{m}, \tau_{9.7 \mu \mathrm{m}}$ (e.g. Shi et al. 2006; Levenson et al. 2007):

$\tau_{9.7 \mu \mathrm{m}}=\ln \left(F_{\text {cont }, 9.7 \mu \mathrm{m}} / F_{9.7 \mu \mathrm{m}}\right)$

where $F_{9.7 \mu \mathrm{m}}$ and $F_{\text {cont,9.7 } \mu \mathrm{m}}$ are the fluxes of the spectra around $9.7 \mu \mathrm{m}$ and its expected continuum, respectively. The apparent depth at $9.7 \mu \mathrm{m} \tau_{9.7} \mu \mathrm{m}$ is positive for absorption silicate features and negative for emission features.

Owing to the complexity of Spitzer spectra, we have used PAHFIT $^{6}$ to obtain $\tau_{9.7 \mu \mathrm{m}}$ except when emission silicate features are detected (see below). PAHFIT is an IDL tool for decomposing Spitzer/IRS spectra of PAH emission sources, with a special emphasis on the careful recovery of ambiguous silicate absorption, and weak, blended dust emission features (Smith et al. 2007). PAHFIT is primarily designed for its use with the full 5-35 $\mu \mathrm{m}$ Spitzer/IRS low-resolution spectra. However, PAHFIT is not able to treat or recover silicate emission features expected to occur in Type-1 AGN, giving $\tau_{9.7 \mu \mathrm{m}}=0$. In these cases we computed $\tau_{9.7 \mu \mathrm{m}}$ by fitting the $9-14 \mu \mathrm{m}$ Spitzer/IRS spectra to a Gaussian profile. This is a general treatment to estimate $\tau_{9.7 \mu \mathrm{m}}$, which has been proven to be a good approximation when compared with PAHFIT (see González-Martín et al. 2013).

Together with the Spitzer/IRS spectra of the sample, we also compiled the $12 \mu \mathrm{m}$ luminosities obtained with ground-based telescopes. These measurements have the advantage of better isolating the nuclear source because they come from images with $\sim 0.3$ arcsec spatial resolution (i.e. a few tenths of parsecs for nearby galaxies). Most of these measurements come

\footnotetext{
6 http://tir.astro.utoledo.edu/jdsmith/research/ pahfit.php
} 

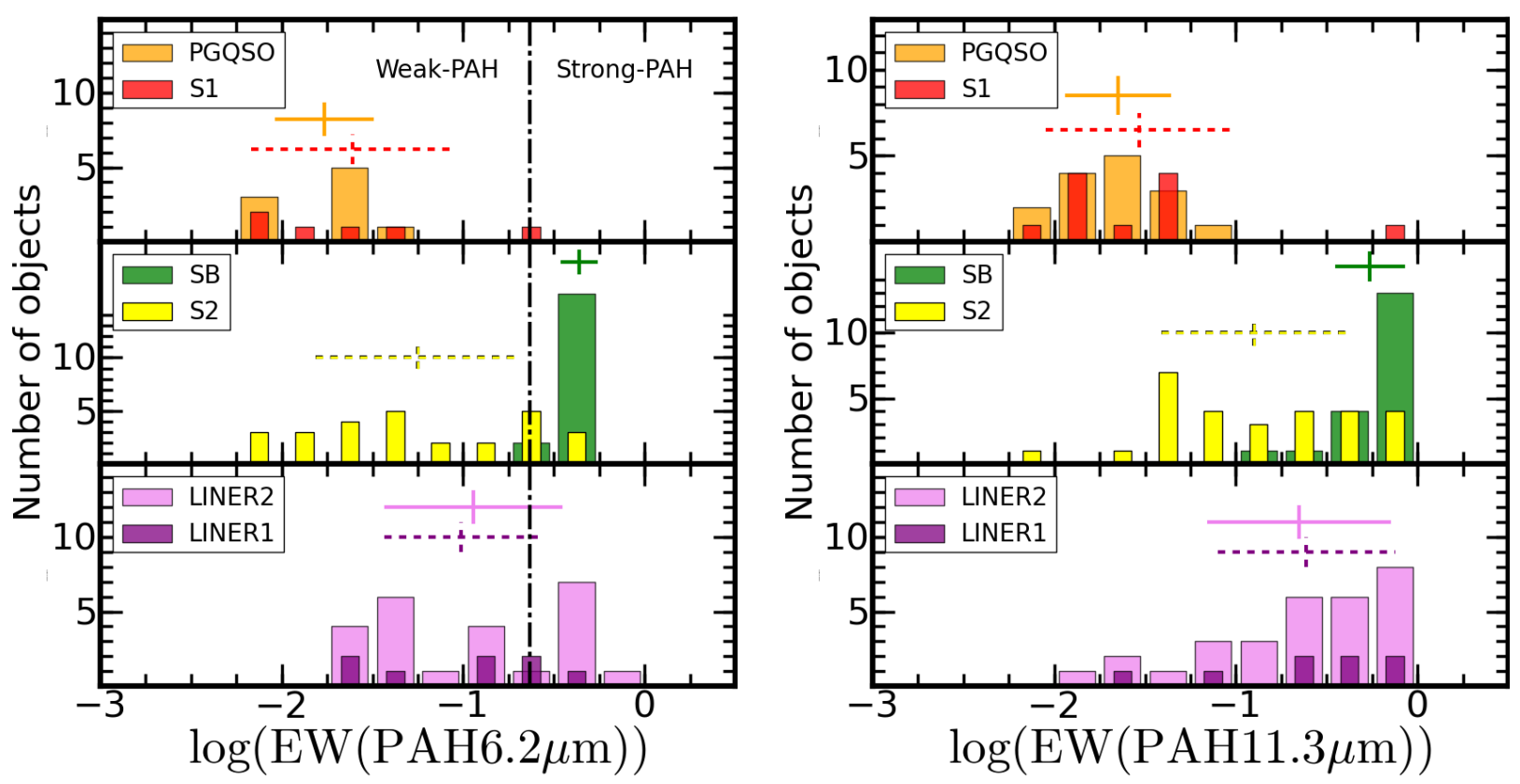

Fig. 1. Histograms of the EW of the PAH at $6.2 \mu \mathrm{m}$ (left) and at $11.3 \mu \mathrm{m}$ (right) for PG QSO (top panel, broad orange bars), S1 (top panel, narrow red bars), SB (middle panel, broad green bars), S2 (middle panel, narrow yellow bars), LINER1 (bottom panel, narrow purple bars), and LINER2 (bottom panel, broad pink bars). The median values and 25th-75th percentile range for each class of object are shown with large crosses (with the same colour code as the histogram) with continuous lines for PGQSO, SB, and LINER2 and with dashed lines for S1, S2, and LINER1. The vertical dot-dashed line shows the limit chosen to divide the sample into strong- and weak-PAHs (see text).

from the catalogue of sub-arcsecond mid-IR images of AGN reported by Asmus et al. (2014). It includes Subaru/COMICS, VLT/VISIR, Gemini/MICHELLE, and Gemini/T-ReCS mid-IR data of 253 objects.

We have also included four LINERs observed with GTC/CanariCam as proprietary data (programme IDs GTC42$12 \mathrm{~B}$ and GTC35-13A). We reduced them uniformly with the RedCan package (González-Martín et al. 2013). We present here their luminosities (marked with asterisks in Col. 5 in Table 1), whereas the full imaging analysis will be presented in a forthcoming paper. All together we have ground-based measurements for 61 out of the 129 sources included in this paper.

Table 1 contains the X-ray luminosities, mid-IR measurements for the Spitzer/IRS spectra, and the $12 \mu \mathrm{m}$ luminosities from Spitzer/IRS and ground-based telescopes when available.

\section{AGN versus starburst contents}

Spitzer has been used to study the largest samples of AGN ever analysed at mid-IR (e.g. Shi et al. 2006; Deo et al. 2007). However, one disadvantage of these data is their relatively low spatial resolution. This makes Spitzer spectra often be contaminated by the host galaxy. It is expected to be particularly relevant for LINERs where the AGN is faint. In this case the non-AGN contribution might be very strong at mid-IR wavelengths, dominating the entire emission (Mason et al. 2012).

Over the past decade, several diagnostics have been proposed to quantify the contribution of star formation and AGN activity to the infrared luminosity. These diagnostics are based on the mid-IR continuum slope, the EW of the PAH features, the ratio of [NeV] (or [OIV]) over [NeII], and the EW of the PAH at 6.2 or $11.3 \mu \mathrm{m}$ versus the $9.7 \mu \mathrm{m}$ optical depth $\tau_{9.7 \mu \mathrm{m}}$ (Genzel et al. 1998; Lutz et al. 1998; Dale et al. 2006; Sturm et al. 2006; Spoon et al. 2007; Baum et al. 2010; Hernán-Caballero \& Hatziminaoglou 2011). In this section we use several diagnostics to separate those Spitzer/IRS spectra that are strongly contaminated by non-AGN emission; however, the SB contribution to the mid-IR spectra does not exclude the presence of an AGN. This is an attempt to determine whether the AGN is dominating the mid-IR spectrum. We have excluded the diagnostics from the analysis based on fine structure emission lines because they are blended with other emission lines at the spectral resolution of these dataset. For more details in these diagnostics, we refer the reader to Dudik et al. (2009), where they studied these emission lines for a large sample of LINERs.

\subsection{PAH features}

The star formation activity correlates with the PAH strength, where SB-dominated galaxies are then expected to show strong PAH features (e.g. Peeters et al. 2004). This well-established correlation has led to the use of PAH strength as a tracer of star formation (e.g. Esquej et al. 2014). PAHs might be destroyed by the presence of an AGN (Genzel et al. 1998; Wu et al. 2009). This is particularly relevant for the PAH feature at $6.2 \mu \mathrm{m}$ that is produced by grains with smaller sizes, and therefore, their destruction near the AGN is more efficient (Diamond-Stanic \& Rieke 2012). However, the $11.3 \mu \mathrm{m}$ might not be suppressed by the AGN (at distances as close as $10 \mathrm{pc}$ ) but diluted when the AGN continuum becomes dominant (Alonso-Herrero et al. 2014; Ramos Almeida et al. 2014). Our aim is to select those spectra where the host galaxy contribution due to star formation does not dominate the mid-IR spectrum. The PAH strength is a good tracer of star formation occurring far away from the AGN, where this destruction or dilution of the PAH features is negligible. Supporting this, the PAH at $11.3 \mu \mathrm{m}$ was negligible in 18 out of the 20 AGN reported by González-Martín et al. (2013) with high-spatial resolution spectra, while their Spitzer spectra showed strong PAH features. Thus, these diagnostics are still useful in our analysis, irrespective of the dilution or suppression of the PAH features near the AGN. 
Figure 1 shows the distributions of the $E W(\mathrm{PAH})$ per class for the PAH features detected at $6.2 \mu \mathrm{m}$ (left) and $11.3 \mu \mathrm{m}$ (right). Upper limits on the undetected lines are not included in these histograms. The median $E W(\mathrm{PAH} 6.2 \mu \mathrm{m})$ for the $\mathrm{SB}$ class is significantly higher than for the S1 and PGQSO classes. Only one $\mathrm{S} 1$ shows an $E W(\mathrm{PAH} 6.2 \mu \mathrm{m})$ consistent with the SB class (NGC 5033). Moreover, only one SB shows a limit on the $E W(\mathrm{PAH} 6.2 \mu \mathrm{m})$ that is consistent with $\mathrm{S} 1 \mathrm{~s}$ or PG QSOs (NGC 3184). The S2 class have objects with $E W(\mathrm{PAH}$ $6.2 \mu \mathrm{m}$ ) overlapping with values found for S1s, PGQSOs, and SBs. LINERs (both LINER1 and LINER2), like S2s, also show $E W(\mathrm{PAH} 6.2 \mu \mathrm{m})$ spreading over a wide range of values. The histogram of $E W(\mathrm{PAH} 11.3 \mu \mathrm{m})$ is similar to that of $E W(\mathrm{PAH}$ $6.2 \mu \mathrm{m})$. However, there is a larger overlapping between the distributions in $E W(\mathrm{PAH} 11.3 \mu \mathrm{m})$ for SBs with S1s and PGQSOs than in the histogram of $E W(\mathrm{PAH} 6.2 \mu \mathrm{m})$. Moreover, the $\mathrm{S} 2$ class overlaps with the SB class for a larger number of objects in $E W(\mathrm{PAH} 11.3 \mu \mathrm{m})$ than for the histogram of $E W(\mathrm{PAH} 6.2 \mu \mathrm{m})$ (15 and 6 objects, respectively).

The PAH feature at $11.3 \mu \mathrm{m}$ might be strongly attenuated by the silicate absorption feature (Brandl et al. 2006). GonzálezMartín et al. (2013) estimated this attenuation to be up to $\sim 40 \%$ of the intrinsic PAH feature at $11.3 \mu \mathrm{m}$ for $\tau_{9.7 \mu \mathrm{m}}=1$. This percentage can be higher for larger $\tau_{9.7 \mu \mathrm{m}}$. The $E W(\mathrm{PAH} 6.2 \mu \mathrm{m})$ might be a better tracer of star formation when the silicate attenuation is large because it is not embedded in the silicate absorption feature. This is clearly seen in Fig. 1, where the overlap of the S2 class (expected to be more attenuated than S1s under the unified model) with the SBs is much higher for the $E W(\mathrm{PAH}$ $11.3 \mu \mathrm{m})$ than for the $E W(\mathrm{PAH} 6.2 \mu \mathrm{m})$. We therefore have chosen $E W(\mathrm{PAH} 6.2 \mu \mathrm{m})$ as a better tracer of the SB-dominance in our sample.

We have defined a limit on $E W($ PAH $6.2 \mu \mathrm{m})$ using the mean value and the standard deviation over this mean value for objects classified as SBs as follows: < $E W($ PAH6.2 $\mu \mathrm{m})>-3 \times \sigma(E W($ PAH6.2 $\mu \mathrm{m}))=0.233 \mu \mathrm{m}$ (i.e. $<\log (E W($ PAH6.2 $\mu \mathrm{m}))>=-0.633)$. This ensures that $99.7 \%$ of SBs show $E W(\mathrm{PAH} 6.2 \mu \mathrm{m})$ above this limit. We note here that the limit would be $E W($ PAH6.2 $\mu \mathrm{m})=0.247$ if the 99th percentile was used. This would give a slightly less restrictive limit ${ }^{7}$. Above (below) this value we classified the objects as strong-PAH (weak-PAH) objects. Six out of the $31 \mathrm{~S} 2 \mathrm{~s}$ are consistent with the strong-PAH category, and ten out of the 40 LINERs are classified within the strong-PAH class. All of them are LINER2s except NGC 1097.

Spoon et al. (2007) present a mid-IR diagnostic of the AGN/ULIRG content based on $\tau_{9.7}$ versus $E W($ PAH $6.2 \mu \mathrm{m})$ (see also Hernán-Caballero \& Hatziminaoglou 2011). The advantage of this diagnostic is that it takes the effects of strong obscuration of the nuclear source into account. They show that galaxies are systematically distributed along two different branches: (1) a horizontal line with $\tau_{9.7}<1$ of continuum AGN-dominated to PAH-dominated spectra and (2) a diagonal line going from deeply obscured (high $\tau_{9.7}$ and low $E W\left(\mathrm{PAH} 6.2 \mu \mathrm{m}\right.$ ) to PAH-dominated spectra (low $\tau_{9.7}$ and high $E W(\mathrm{PAH} 6.2 \mu \mathrm{m})$. Seyferts and QSOs are found exclusively on the horizontal branch with $\tau_{9.7}<1$. The large majority of LIRGs and ULIRGs in Spoon et al. (2007) are located in the diagonal line. Starbursts are placed at the end of the two branches, with large $E W(\mathrm{PAH} 6.2 \mu \mathrm{m})$ and $\tau_{9.7}<1$. They argue that these two

\footnotetext{
7 Only two objects will be included in the weak-PAH class if the limit is set to $E W(\mathrm{PAH} 6.2 \mu \mathrm{m})=0.247$ compared to those obtained using $E W($ PAH6.2 $\mu \mathrm{m})>0.233$, namely NGC 7130 and NGC 3367.
}

branches reflect a fundamental difference in the dust geometry in the two sets of sources. The horizontal branch could have a clumpy structure while the diagonal might be smooth.

Figure 2 shows $\tau_{9.7}$ versus $E W(\mathrm{PAH} 6.2 \mu \mathrm{m})$ for $\mathrm{SBs}, \mathrm{S} 1 \mathrm{~s}$, and PGQSOs in the left-hand panel and LINERs and S2s in the right-hand panel. PGQSOs and S1s $\left(\tau_{9.7}<1\right.$ and $E W(\mathrm{PAH}$ $6.2 \mu \mathrm{m})<0.228 \mu \mathrm{m})$ are clearly distinguished from SBs $(E W(\mathrm{PAH} 6.2 \mu \mathrm{m})>0.233 \mu \mathrm{m})$. This result is fully consistent with what is reported by Spoon et al. (2007). Our diagram shows very few nuclei with deep silicate features and weak PAH features. This is also found by Spoon et al. (2007) with only eight over the 160 objects in their sample belonging to this category. We have tested the use of the $E W(\mathrm{PAH} 11.3 \mu \mathrm{m})$ instead of $E W(\mathrm{PAH} 6.2 \mu \mathrm{m})$ in this diagram, finding a similar result. This has already been reported by Hernán-Caballero \& Hatziminaoglou (2011) in a large sample of Spitzer/IRS spectra.

There is a maximum $\tau_{9.7}$ expected under the predictions of the clumpy torus models for AGN. Higher values of $\tau_{9.7}$ can be interpreted as significant contamination from the host galaxy (Alonso-Herrero et al. 2011; González-Martín et al. 2013). To investigate this issue, we computed $\tau_{9.7}$ using a set of models within the libraries of CLUMPY ${ }^{8}$. These consist of a set of spectral energy distributions (SEDs) using the AGN clumpy torus emission described by Nenkova et al. (2008). The parameter ranges chosen are those reported by González-Martín et al. (2013). We have downloaded the SEDs for a width of the toroidal distribution $\sigma=45^{\circ}$, a ratio between the outer and the inner radii of the torus $r_{\text {out }} / r_{\text {int }}=200$, an exponential slope of the radial distribution of clouds $q=2$, an optical extinction of the clouds in the range $\tau_{\mathrm{V}}=5-150$, and a number of clouds along the equator of the torus $N_{\mathrm{o}}=2-20$ clouds. The number of clouds along the LOS, $N$, depends on the inclination angle, $i$, as $N=N_{\mathrm{o}} \mathrm{e}^{-i^{2} / \sigma^{2}}$. We refer the reader to González-Martín et al. (2013) for details on the selection of these parameters and to Nenkova et al. (2008) for the details on the modelling.

We computed $\tau_{9.7}$ for these SEDs using the same methodology as for the Spitzer/IRS spectra reported here. Figure 2 shows the minimum and maximum $\tau_{9.7}$ found using these models $\left(-1.13<\tau_{9.7}<1.25\right)$. Thus, objects with $\tau_{9.7}>1.25$ are not expected under any clumpy torus model. We use $\tau_{9.7}>$ 1.25 to classify an object as a deep silicate. We also show the range of $\tau_{9.7}$ expected for face-on AGN assuming $i=0^{\circ}$ $\left(-0.96<\tau_{9.7}<0.62\right)$ and for edge-on AGN assuming $i=90^{\circ}$ $\left(-0.86<\tau_{9.7}<1.25\right)$. S1s are naturally explained within the range of values of $\tau_{9.7}$ expected under the clumpy torus models. S2s tend to show larger $\tau_{9.7}$ than S1s. Only two S2s are out of the expected range with clumpy torus models. Only two SBs and two LINERs show $\tau_{9.7}>1.25$. Following this diagram, we have divided our sample into three categories:

- Deep-silicate: Strength of the silicate feature above the maximum explained by clumpy models (i.e. $\tau_{9.7}>1.25$ ) regardless of $E W(\mathrm{PAH} 6.2 \mu \mathrm{m})$.

- Strong-PAH: $\tau_{9.7}<1.25$ and $E W(\mathrm{PAH} 6.2 \mu \mathrm{m})>0.233 \mu \mathrm{m}$.

- Weak-PAH: $\tau_{9.7}<1.25$ and $E W($ PAH $6.2 \mu \mathrm{m})<0.233 \mu \mathrm{m}$.

This classification is included in Table 1. Starbursts are located mostly in the region of strong-PAH. Two of them, though, populate the area of deep silicates. PGQSOs and S1s are placed in the region of weak PAHs ${ }^{9}$.

\footnotetext{
8 http://www . pa.uky.edu/clumpy/

9 The only exception is NGC 5033, which is located in the region of the diagram of strong PAHs.
} 


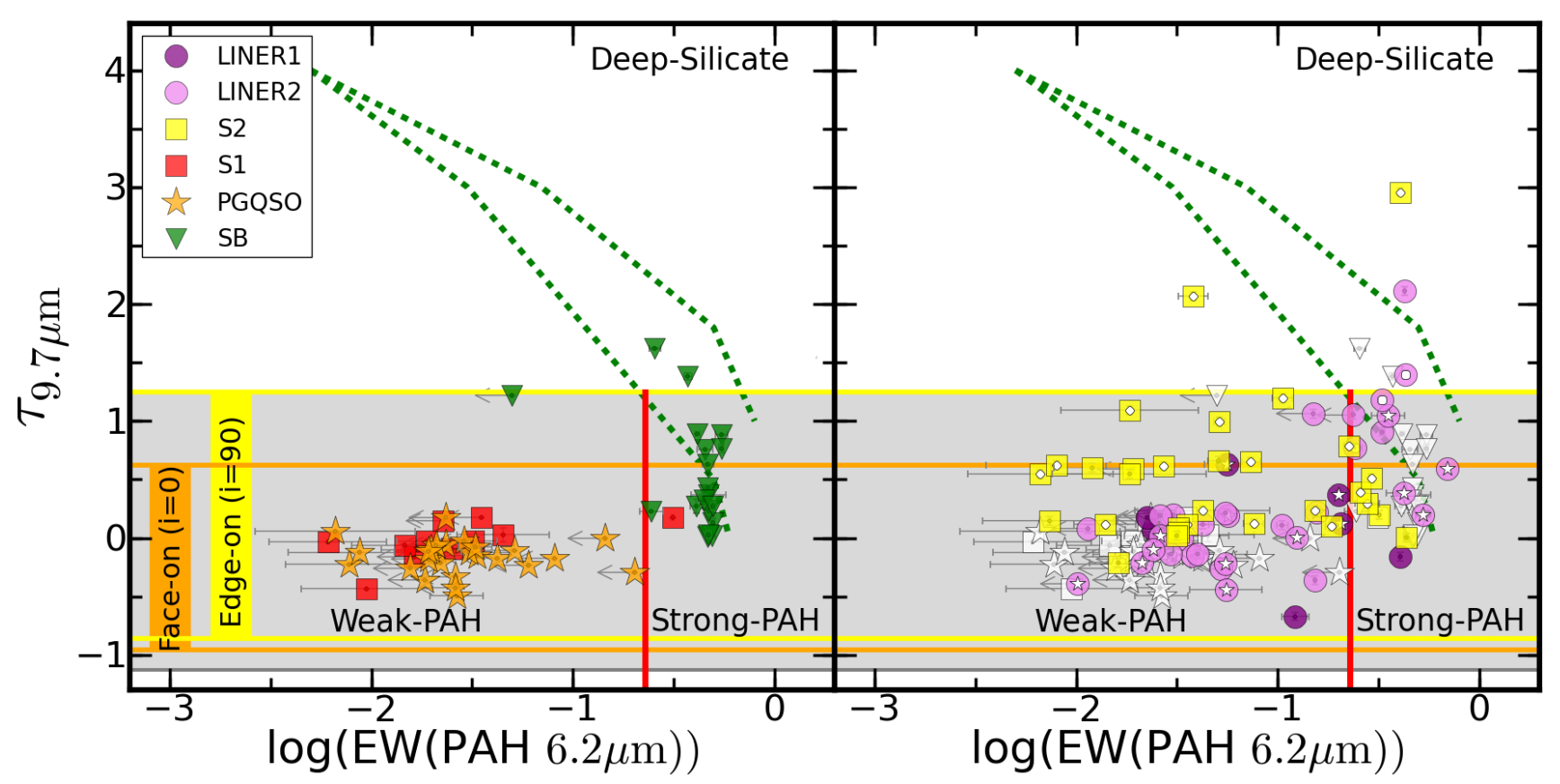

Fig. 2. Silicate apparent depth at $9.7 \mu \mathrm{m}, \tau_{9.7}$, versus the EW of PAH at $6.2 \mu \mathrm{m}, E W(\mathrm{PAH} 6.2 \mu \mathrm{m}$ ), for PGQSOs, S1s, and SBs (left) and for LINERs and S2s, (right). LINER1s, LINER2s, S1s, S2s, PG-QSOs, and SBs are shown with purple and pink circles, red and yellow squares, orange stars, and green upside-down triangles, respectively. White circles and white stars denote known Compton-thick sources and Comptonthick candidates, respectively. The error bars in $\tau_{9.7}$ are always within the size of the symbol. In the right panel we also include PG QSOs, S1s, and SBs with white symbols for comparison purposes. Green-dotted lines indicate the diagonal branch found by Spoon et al. (2007) for ULIRGs and SBs. The shadowed (grey) area shows the range of values for $\tau_{9.7}$ that could be explained with clumpy models (Nenkova et al. 2008). We also use orange and yellow lines to show the expected range of values for $\tau_{9.7}$ in face-on AGN (assuming $i=0^{\circ}$ ) and in edge-on AGN (assuming $i=90^{\circ}$ ) using the models described by Nenkova et al. (2008) (see text). The short red vertical line shows $E W$ (PAH6.2 $\mu \mathrm{m})=0.228 \mu \mathrm{m}$, which divides into weak and strong PAHs.

S2s tend to show larger $\tau_{9.7}$ than S1s, as expected under the unified model of AGN. Similar to S1s and PGQSOs, most S2s are in the region of weak PAH. Only two of them are within the area of deep silicates, and five of them are within the area of strong PAHs. Thus, according to this diagram, only seven out of the 31 S2s show signs of host-galaxy contamination at mid-IR.

LINERs mostly populate the area of weak PAHs. All the LINER1s but NGC 1097 are in this area of the diagram. Among LINER2s, two are in the region of deep silicates and nine in the strong-PAHs area. If LINERs in the weak-PAH area of the diagram are considered as AGN-dominated at mid-IR, then 30 out of the 41 are AGN-dominated at mid-IR.

\subsection{Steepness of the mid-IR spectra}

The steepness of the mid-IR spectra characterises the relative contribution of warm and cool dust to the mid-IR (Baum et al. 2010). We refer here to the steepness at the spatial resolutions of the Spitzer/IRS spectra (i.e. kpc scales), while on smaller scales (below $100 \mathrm{pc}$ scales obtained with ground-based instruments), the mid-IR emission is expected to be dominated from dust heated by the AGN (Hönig et al. 2011; Ramos Almeida et al. 2011). It has proven to be a good indicator of the SB content given its correlation with $E W(\mathrm{PAH} 11.3 \mu \mathrm{m})$ (e.g. Wu et al. 2009; Weedman et al. 2005; Brandl et al. 2006; LaMassa et al. 2012). This steepness has been defined in several bands by different authors; e.g. 20-30 $\mu \mathrm{m}$ (Baum et al. 2010; Weedman et al. 2005) or 15-30 $\mu \mathrm{m}$ (Brandl et al. 2006; Wu et al. 2009; Nardini et al. 2008). We present the 20 and $30 \mu \mathrm{m}$ luminosities to produce an estimate of the steepness of the mid-IR spectra in our sample.
Figure 3 shows the histogram of such steepness, expressed as $\log \left(v L_{v}(20 \mu \mathrm{m}) / v L_{v}(30 \mu \mathrm{m})\right.$ ) (see also Table 1). PGQSOs and S1s tend to show higher values of $\log \left(v L_{v}(20 \mu \mathrm{m}) / v L_{v}(30 \mu \mathrm{m})\right)$ than SBs. However, these two distributions overlap in the range $-0.25<\log \left(v L_{v}(20 \mu \mathrm{m}) / v L_{v}(30 \mu \mathrm{m})\right)<0$. S2s and LINERs show a wide range of $\log \left(v L_{v}(20 \mu \mathrm{m}) / v L_{v}(30 \mu \mathrm{m})\right)$, overlapping with S1s, PGQSOs, and SBs, although S1s and PGQSOs distributions are skewed toward the higher values of $\log \left(v L_{\nu}(20 \mu \mathrm{m}) / v L_{v}(30 \mu \mathrm{m})\right)$. Therefore, this ratio itself is not as good a tracer of AGN dominance as it is of $E W(\mathrm{PAH}$ $6.2 \mu \mathrm{m}$ ) (see previous section). Although in theory it is a good tracer of the contribution of warm and cool dust to the mid-IR; in practice, some SB-dominated spectra can have a $\log \left(v L_{v}(20 \mu \mathrm{m}) / v L_{v}(30 \mu \mathrm{m})\right)$ ratio consistent with those of AGN.

Following the same idea as in the previous section, we computed $\log \left(\nu L_{\nu}(20 \mu \mathrm{m}) / \nu L_{\nu}(30 \mu \mathrm{m})\right)$ of theoretical models obtained with the Clumpy libraries. Coloured areas (Fig. 3) show the range of steepness expected from these models for face-on (assuming $i=0$ ) and edge-on (assuming $i=90$ ) tori, respectively. The expected range of values found for the models of Type-1 AGN is almost identical to the range of values found for PGQSOs and S1s. Moreover, the steepness of the spectra in the model of Type-2 AGN is expected to include the same range of values as S1s but extended towards lower values. Thus, Type-2 AGN are expected to show steeper spectra than Type-1 AGN. This agrees with our results, where S2s tend to show lower $\log \left(v L_{\nu}(20 \mu \mathrm{m}) / v L_{v}(30 \mu \mathrm{m})\right)$ than S1s. Seven out of the $20 \mathrm{SBs}$ have a steepness that is fully consistent with the model of Type-2 AGN. All the objects below the minimum steepness predicted for Type-2 AGN 


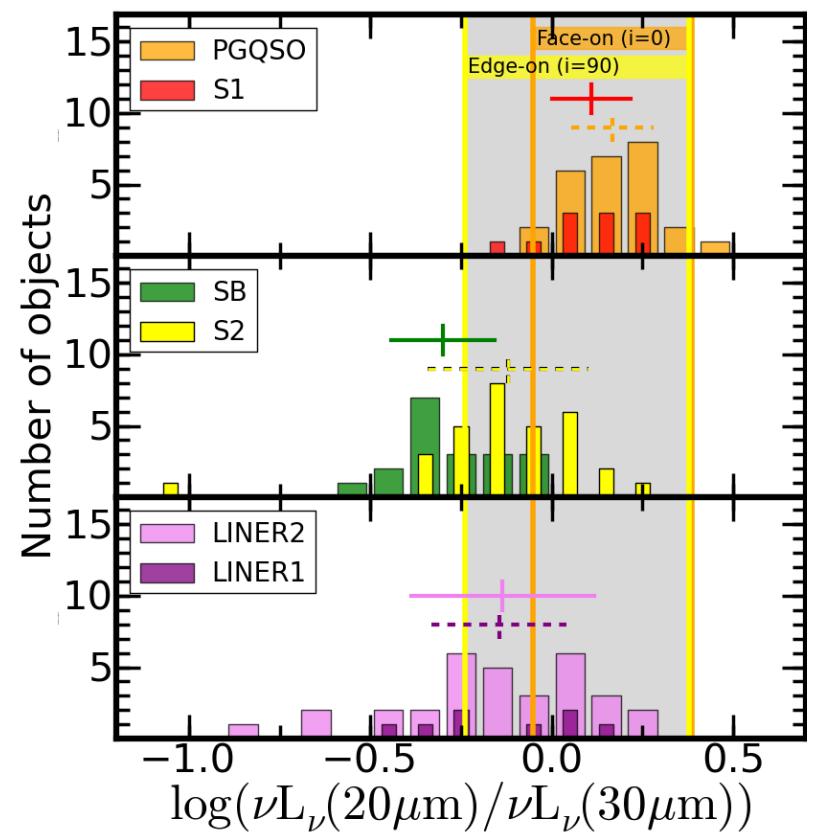

Fig. 3. Histograms of $\log \left(v L_{v}(20 \mu \mathrm{m}) / v L_{v}(30 \mu \mathrm{m})\right)$ for PGQSO (top panel, broad orange bars), S1 (top panel, narrow red bars), SB (middle panel, broad green bars), S2 (middle panel, narrow yellow bars), LINER1 (bottom panel, narrow purple bars), and LINER2 (bottom panel, broad pink bars). The mean values and one standard deviation over the mean for each class of objects are shown with large crosses (with the same colour code as the histogram), continuous lines for PG QSO, SB, and LINER2, and dashed lines for S1, S2, and LINER1. The grey area of the plot shows the range of values expected for AGN according to the models given by Nenkova et al. (2008). The orange and yellow vertical lines show the same ranges but for inclination angles of $i=0^{\circ}$ and $i=90^{\circ}$, assuming that these values are representative of face-on and an edge-on AGN (see text).

by the Clumpy libraries can be considered as SB-dominated $\left(\log \left(v L_{v}(20 \mu \mathrm{m}) / v L_{v}(30 \mu \mathrm{m})\right)=-0.24\right)$. However, there are SBs above that limit. Thus, $\log \left(v L_{v}(20 \mu \mathrm{m}) / v L_{v}(30 \mu \mathrm{m})\right)<-0.24$ indicates that the spectrum is SB-dominated, but we cannot discard that spectra showing $\log \left(v L_{v}(20 \mu \mathrm{m}) / v L_{v}(30 \mu \mathrm{m})\right)>-0.24$ might also be SB-dominated.

We have classified those showing $\log \left(v L_{v}(20 \mu \mathrm{m}) / v L_{v}\right.$ $(30 \mu \mathrm{m}))<-0.24$ as SB-dominated spectra. Among the S2s, six objects are therefore SB-dominated, three of them already classified as SB-dominated according to the strengths of the PAHs and silicate features. Combining both methods together, ten out of the $31 \mathrm{~S} 2 \mathrm{~s}(32 \%)$ are SB-dominated.

Thirteen out of the 41 LINERs show $\log \left(v L_{v}(20 \mu \mathrm{m}) /\right.$ $\left.v L_{v}(30 \mu \mathrm{m})\right)<-0.24$. Two are LINER1s) and the remaining 11 are LINER2s. Among them, eight were already classified as SB-dominated using the strengths of PAHs and silicate features. Interestingly, only two LINERs classified as SB-dominated with the diagram seen in Fig. 2 are not SB-dominated using the steepness of the spectra. Fifteen out of the 40 LINERs (37.5\%) show signatures of being SB-dominated once the two methods presented in this section are considered together. The fraction of SB-dominated LINERs is similar to that of S2s.

\subsection{Goodness of the methodology to trace nuclear properties}

We have considered a mid-IR spectrum as AGN-dominated if it obeys three criteria: $E W(\mathrm{PAH} 6.2 \mu \mathrm{m})<0.233 \mu \mathrm{m}, \tau_{9.7}<1.25$, and $\log \left(v L_{v}(20 \mu \mathrm{m}) / v L_{v}(30 \mu \mathrm{m})\right)>-0.24$. To study the goodness of this method for selecting AGN-dominated Spitzer/IRS spectra, we have plotted in Fig. 4 the $12 \mu \mathrm{m}$ flux for the Spitzer/IRS spectra versus the same quantity for ground-based measurements for the 63 objects for which these measurements are available (see Sect. 3). Ground-based and Spitzer/IRS $12 \mu \mathrm{m}$ fluxes show a linear relation, although the dispersion is high (Pearson correlation coefficient of $r=0.64, P($ null $)=1.6 \times$ $10^{-7}$ ). Moreover, the slope of the best linear fit (left panel of Fig. 4) is flatter than the one-to-one relation.

Most of the Spitzer/IRS spectra with larger $12 \mu \mathrm{m}$ flux than those from ground-based measurements are SB-dominated according to the $E W(\mathrm{PAH} 6.2 \mu \mathrm{m})$ method (see middle panel of Fig. 4). However, not all the SB-dominated Spitzer/IRS objects show a $12 \mu \mathrm{m}$ flux excess in the Spitzer/IRS spectra compared to the ground-based measurements. We rule out the explanation of a distance effect in which more distant objects might include more SB-contribution in the nuclear spectra, because all our objects are nearby, and no particular trend is found when comparing SB- and AGN-dominated sources. Alternatively, this result might have two explanations: (1) our method of selecting SB-dominated spectra is too restrictive and could include SBdominated spectra that are actually AGN-dominated at $12 \mu \mathrm{m}$; and (2) the Spitzer/IRS spectra do not contain extra emission compared to the ground-based measurements and both trace a nuclear SB-dominated spectrum.

If we select only AGN-dominated Spitzer spectra, the correlation between these two quantities improves (see Fig. 4, right panel) with a Pearson correlation coefficient of $r=0.88$ $\left(P(\right.$ null $\left.)=5.7 \times 10^{-14}\right)$. The linear fit to the AGN-dominated sources (Fig. 4, right panel) is very close to the one-to-one relation (Fig. 4, right panel). The only two outliers are NGC 4594 and NGC 5866. Thus, when the AGN-dominated spectra are selected, the nuclear $12 \mu \mathrm{m}$ flux obtained with ground-based data is very close to the value obtained by the Spitzer/IRS spectra. This reinforces our methodology as a good tool for isolating AGNdominated mid-IR Spitzer/IRS spectra.

\section{Average spectra}

Figure 5 shows the average spectrum for each class of objects. These average spectra were computed after normalising them to the flux at $15 \mu \mathrm{m}$. The shaded regions show the standard deviation over the average spectrum. We computed the mean value for S2s and LINERs according to our mid-IR classification (see previous section) as AGN-dominated and SB-dominated (left and right panels, respectively).

The average spectra of S1s and PGQSOs show similar shapes, with the silicate feature in emission and similar steepness of the spectra (see left panel in Fig. 5). The relative differences between these two classes are an enhancement of the strength of the silicate feature in emission for PGQSOs compared to S1s and a slightly steeper spectrum for S1s compared to PGQSOs. The average SB spectrum is very different to that of S1s or PGQSOs (see right panel of Fig. 5). The main differences are strong PAH features, a steep spectrum, and deep silicate absorption features. Moreover, the classical lines associated with AGN emission, such as [Ne V] at $14.3 \mu \mathrm{m}$ and $24.3 \mu \mathrm{m}$ or [O IV] at $25.9 \mu \mathrm{m}$, are clearly detected in the average spectra of PG QSOs and S1s but are absent in the average SB spectrum. The association of these lines with AGN emission have been questioned, because some are found in some SB galaxies (see Pereira-Santaella et al. 2010). 


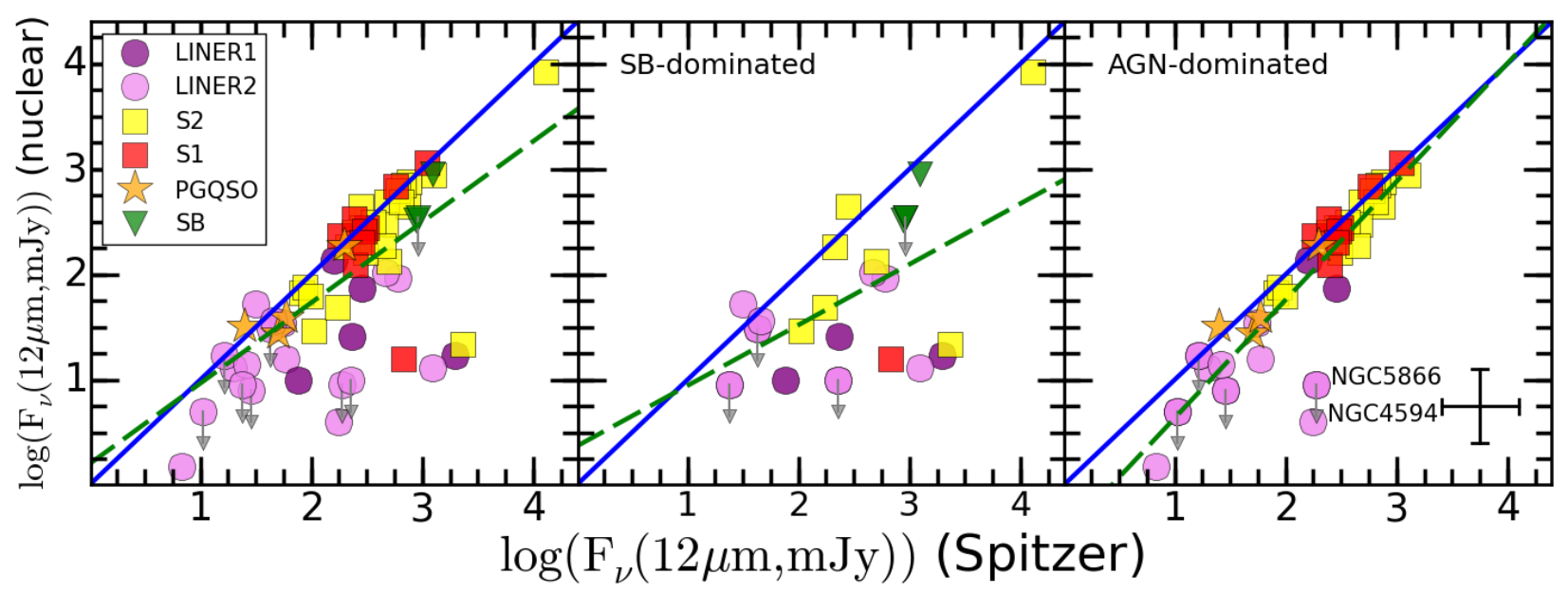

Fig. 4. $12 \mu \mathrm{m}$ flux obtained with ground-based telescopes (nuclear) versus $12 \mu \mathrm{m}$ flux obtained with the Spitzer/IRS spectra. Both quantities are shown on log scales. A cross is shown to illustrate the error bars in these measurements (see Sect. 3). The left panel shows the results for the entire sample of objects with ground-based telescopes, the middle panel shows the objects classified as SB-dominated, and the right panel shows only objects with Spitzer/IRS spectra classified as AGN-dominated in this work (see text). The blue continuous line represents the one-to-one relation and the green dashed line the best linear relation.
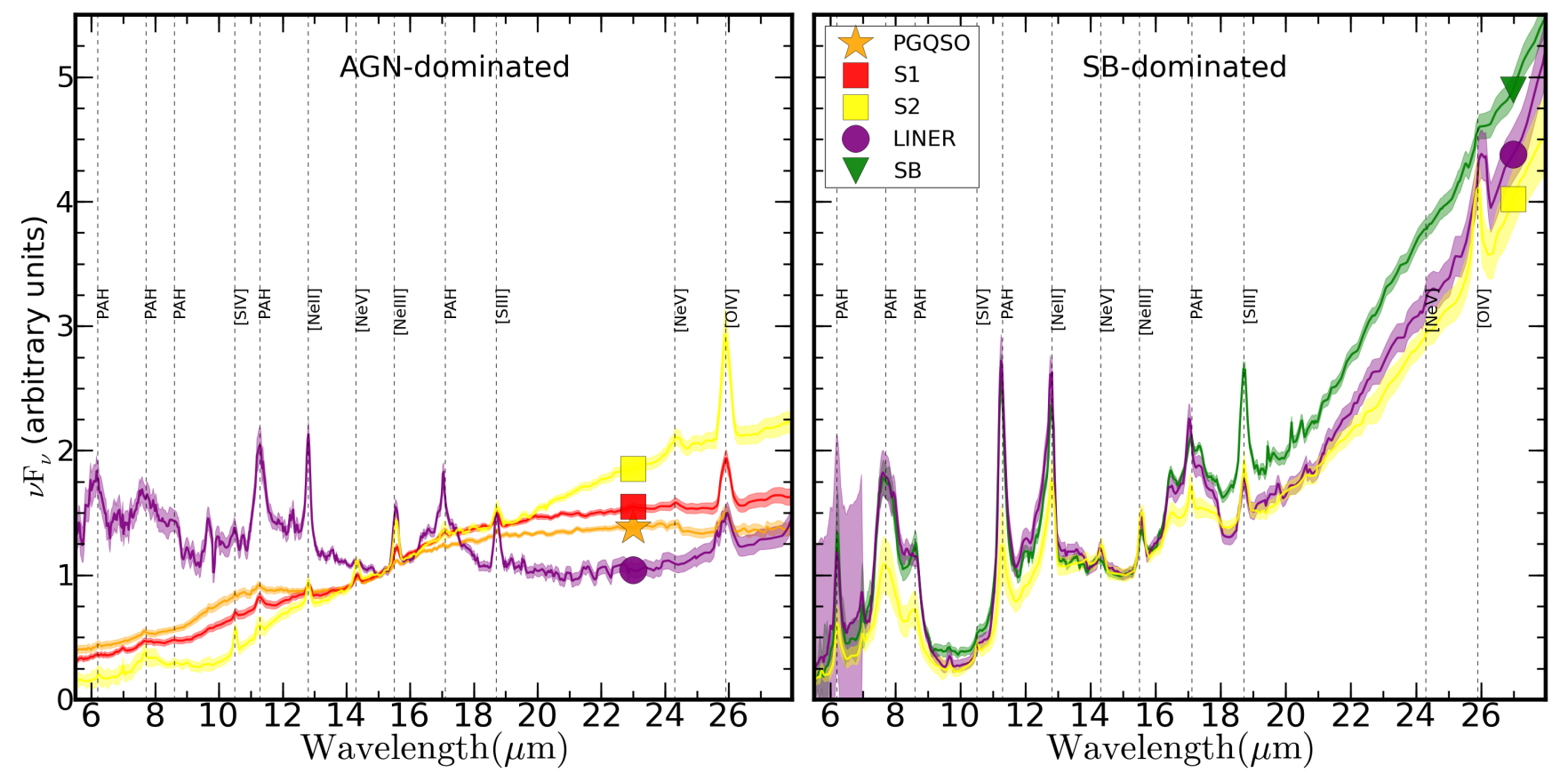

Fig. 5. Left: average spectra for PG QSOs (orange), S1s (red), AGN-dominated S2s (yellow), and AGN-dominated LINERs (purple). Right: average spectra for SBs (green), SB-dominated S2s (yellow), and SB-dominated LINERs (purple). We also show one standard deviation as a shaded region using the same colours. Each spectrum is also marked with different symbols at $27 \mu \mathrm{m}$ for clarity of the plot: PGQSOs (orange star), S1s (red square), SBs (green triangle), S2s (yellow square), and LINERs (purple circle). The average spectra are scaled to the flux at $15 \mu \mathrm{m}$.

The average SB-dominated spectrum for S2s and LINERs (right panel of Fig. 5) are very similar to that of the SBs. The average AGN-dominated (left panel in Fig. 5) and SB-dominated (right panel in Fig. 5) spectra for S2s and LINERs are clearly different. This supports our method as a good diagnostic of SBdominated mid-IR spectra. This was also suggested by AlonsoHerrero et al. (2014), finding that the Spitzer spectra of S1s and S2s are only similar if spectra with deep absorption silicate features are excluded from the analysis. The [Ne V] at $14.3 \mu \mathrm{m}$ and [O IV] at $25.9 \mu \mathrm{m}$ emission lines are clearly seen in the average spectra of SB-dominated S2s and LINERs. This was already shown by Dudik et al. (2009), who find these lines in a large fraction of LINERs. Thus, the average SB-dominated spectra of LINERs and S2s might still show mid-IR signatures of AGN nature, although the overall mid-IR spectra is not dominated by the AGN.

The average S2 AGN-dominated spectrum (left panel of Fig. 5) does not mimic S1s or PG QSOs. This average spectrum is steeper than those of S1s or PGQSOs. It also shows the silicate features in absorption, while S1s and PG QSOs show an average spectra with silicate features in emission. This is expected since the silicate feature at $9.7 \mu \mathrm{m}$ and $18 \mu \mathrm{m}$ are predicted to be in emission for Type-1 AGN and in absorption for Type-2 AGN (Nenkova et al. 2008). These predictions have already been 
confirmed by observations (e.g. Shi et al. 2006). On the similarities, the average (AGN-dominated) S2 spectrum shows the presence of [Ne V] at 14.3 and $24.3 \mu \mathrm{m}$ and [O IV] at $25.9 \mu \mathrm{m}$ emission lines.

The average AGN-dominated LINER spectrum (left panel of Fig. 5) can be clearly distinguished from PG QSOs, S1s, S2s, and SBs. The main characteristic of this average spectrum is the rather flat continuum over the full 6-28 $\mu \mathrm{m}$ wavelength range. Moreover, it shows strong PAH features at $11.3 \mu \mathrm{m}$ and $17 \mu \mathrm{m}$. The [O IV] at $25.9 \mu \mathrm{m}$ emission line is prominent as in $\mathrm{S} 1 \mathrm{~s}$, PGQSOs, and S2s. However, the [Ne V] at $14.3 \mu \mathrm{m}$ emission line is clearly undetected as is the $[\mathrm{Ne} \mathrm{V}]$ at $24.3 \mu \mathrm{m}$. Below $20 \mu \mathrm{m}$ this spectrum resembles that of SBs. However, it can be clearly distinguished from SBs because the average spectrum of LINERs do not show a steep spectrum, and it lacks the silicate absorption features seen in SBs. Moreover, LINERs also show the [O IV] at $25.9 \mu \mathrm{m}$ that the SBs do not show.

As explained in the introduction, the LINERs are a heterogenous family of objects. To study the subclasses of LINERs, Fig. 6 shows the average spectra for several subclassifications of LINERs. These average spectra were computed including only AGN-dominated spectra.

The average spectrum of objects optically classified as LINER1s (panel a in Fig. 6) is steeper than that of LINER2s (panel a). In fact, the average spectrum of Type-1 LINERs is consistent with that of AGN-dominated S2s. However, the dispersion in the average spectrum of LINER1s is quite large because, amongst the five AGN-dominated LINERs1, NGC 4450 shows a flat spectrum that is not consistent with the average trend for this class.

We have also classified the AGN-dominated LINERs into two classes attending to their $L_{\mathrm{X}}(2-10 \mathrm{keV})$ : bright LINERs for objects with $L_{\mathrm{X}}(2-10 \mathrm{keV})>10^{41} \mathrm{erg} \mathrm{s}^{-1}$ and faint LINERs for those with X-ray luminosities below that limit. This is the limit where the torus is expected to disappear at a bolometric luminosity of $L_{\mathrm{bol}} \sim 10^{42} \mathrm{erg} \mathrm{s}^{-1}$ (Elitzur \& Shlosman 2006) ${ }^{10}$. Amongst the AGN-dominated LINERs, seven are classified as bright LINERs and 18 as faint LINERs. The resulting average spectra of bright and faint LINERs (panel b of Fig. 6) are quite similar to that of LINER1s and LINER2s. Thus, bright LINERs show a steeper spectrum compared to faint LINERs, compatible with S1s. The dispersion of the average spectrum of bright LINERs is much lower than that of LINER1s. We investigated whether a different morphology of the host galaxy for bright and faint LINERs could produce the differences in their average mid-infrared spectra. Interestingly, NGC 4450 is the only AGNdominated Type-1 LINER hosted in a late-type galaxy. Only four (out of the 18) faint LINERs and 1 (out of the 7) bright LINERs are hosted in late type galaxies (i.e. $t>1$ ). This lack of latetype galaxies hosting LINERs is expected since they are usually hosted in early-type galaxies (Carrillo et al. 1999). Thus, we have not found any particular tendency in their host-galaxy morphology. Indeed, the average spectrum of faint LINERs hosted in late-type galaxies is consistent with the average spectrum of faint LINERs (and with the average spectrum of faint LINERs hosted in early-type galaxies).

Spectra in panel c of Fig. 6 show the average spectra for Compton-thin and Compton-thick candidates reported by

\footnotetext{
${ }^{10}$ This assumes a conversion between the X-ray luminosity and the bolometric luminosity of $L_{\mathrm{bol}} \simeq 10 \times L_{\mathrm{X}}(2-10 \mathrm{keV})$ (Ho 2008). This conversion factor is the most conservative value we have found (Ho 2009). Any higher value could include more objects as bright LINERs (see also the discussion).
}

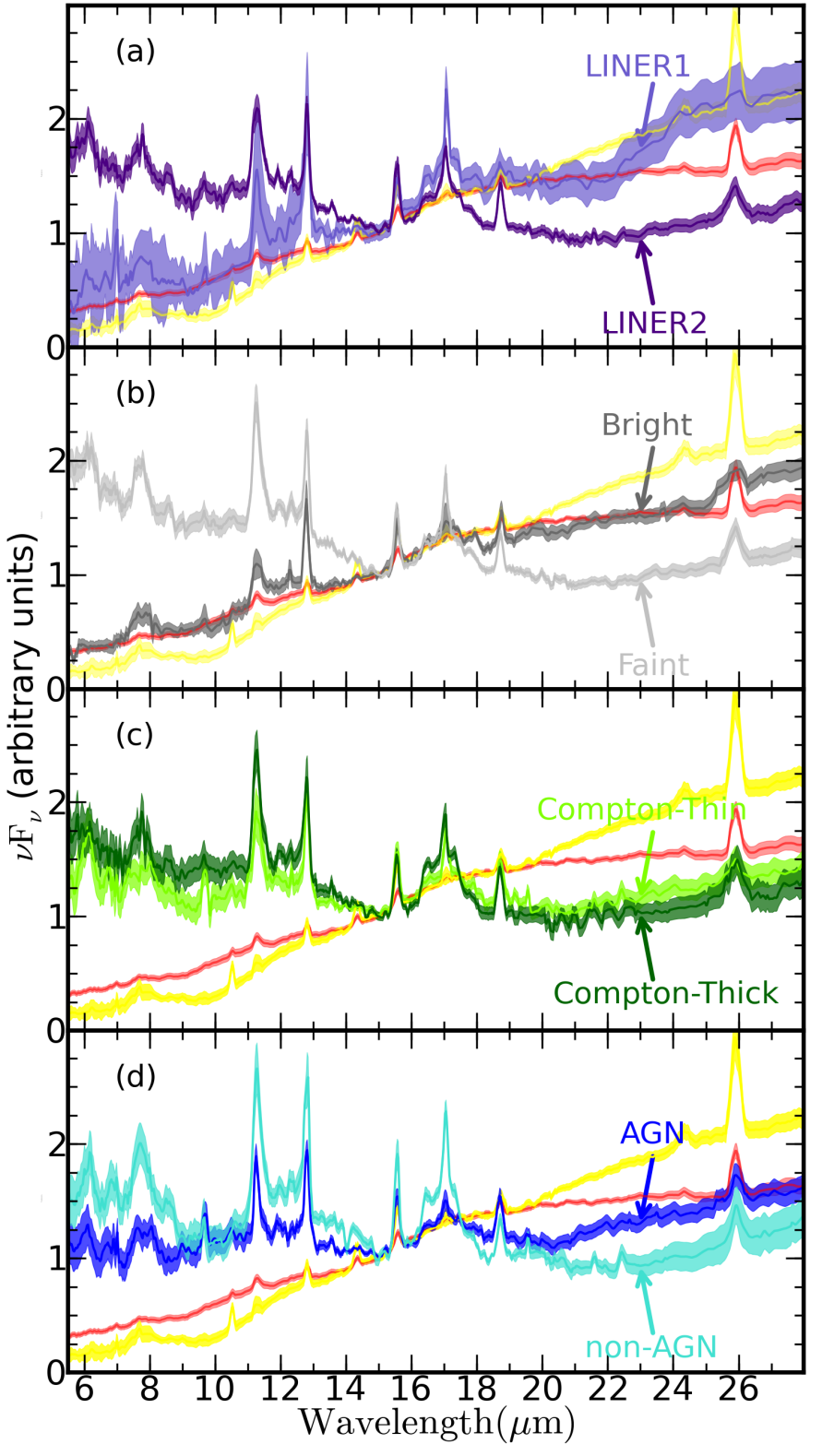

Fig. 6. Average spectra of AGN-dominated LINERs according to different subclassifications. From top to bottom: a) LINER1s (light magenta) and LINER2s (dark magenta); b) bright LINERs (dark grey) and faint LINERs (light grey); c) Compton-thin (light green) and Compton-thick candidates (dark green); d) objects classified at X-rays as AGN (dark blue) and non-AGN candidates (cyan). The average spectra for S1s (red) and AGN-dominated S2s (yellow) are also shown for comparison purposes.

González-Martín et al. (2009b). Compton-thick sources are expected to be so obscured $\left(N_{\mathrm{H}}>1.5 \times 10^{25} \mathrm{~cm}^{-2}\right)$ that the bulk of the X-ray continuum emission of the AGN is only seen above $10 \mathrm{keV}$. Sources were classified according to the ratio between the fluxes of $[\mathrm{O}$ III $] \lambda 5007 \AA$ and the $2-10 \mathrm{keV}$ fluxes, the $\mathrm{EW}$ of the $\mathrm{FeK} \alpha$ emission line, and the hard X-ray slope of the spectrum. Sixteen of them were classified as Compton-thin LINERs, and nine as Compton-thick candidates. We do not find any significant differences between the average mid-IR spectra of Compton-thin and Compton-thick candidates.

González-Martín et al. (2009a) classified 22 out of the 25 AGN-dominated LINERs at X-rays: 16 AGN candidates 


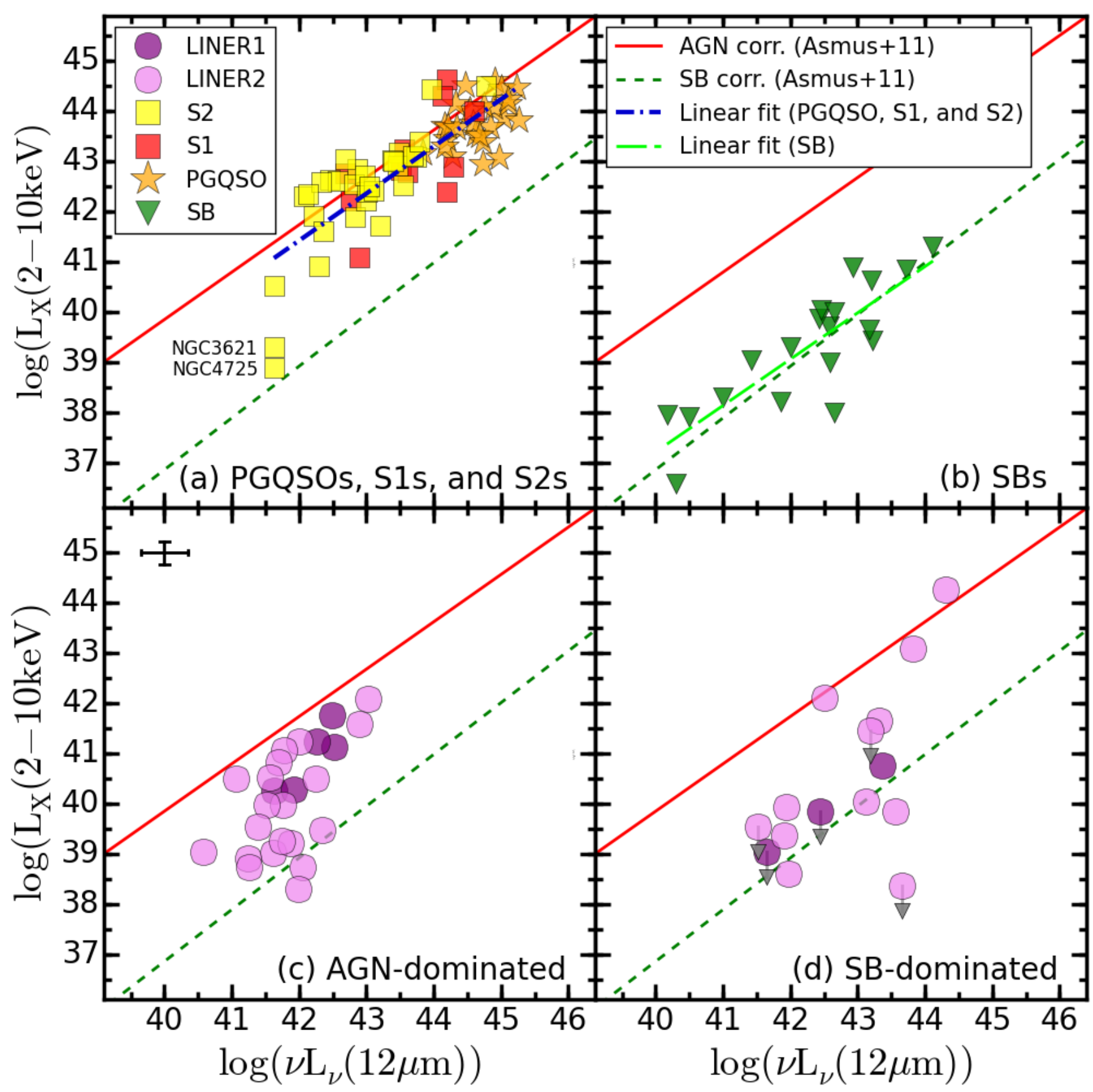

Fig. 7. 2-10 keV luminosity versus the $12 \mu \mathrm{m}$ luminosity, both on logarithmic scale for PGQSOs, S1s and S2s a), SBs b), AGN-dominated LINERs c) and SB-dominated LINERs d). The continuous red and dashed-green lines show the best fit correlations for AGN and SBs, respectively, reported by Asmus et al. (2011). The typical error for these measurements is shown as a cross in the top left corner of panel c). Errors for the X-ray luminosity are estimated as $10 \%$ of its value. The dot-dashed blue line and long-dashed light green line show the linear fit for PG QSOs, S1s, and S2s and for SBs, respectively. Grey arrows mark objects with reported upper limits on the X-ray luminosity.

and 6 non-AGN candidates. Both average spectra are quite flat (panel d of Fig. 6). Interestingly, PAH features and [Ne II] at $12.8 \mu \mathrm{m}$ strengths are larger for non-AGN candidates than for AGN candidates, suggesting the dominance of the host galaxy contribution.

\section{X-ray versus mid-IR luminosities}

In AGN, the X-ray emission is thought to originate in the innermost regions of the accretion flow by Comptonisation processes. The mid-IR emission is generally dominated by thermal emission by dust at parsec-scale distances from the SMBH.
The tight correlation found between the X-ray and mid-IR emission of AGN supports this physical connection, regardless of the Seyfert type (Krabbe et al. 2001; Gandhi et al. 2009; Levenson et al. 2009). Furthermore, SB nuclei also show a relation between X-ray and mid-IR luminosities, but it is offset when compared to that of AGN (Asmus et al. 2011). The study of this correlation could shed light on the dominant process involved in LINERs.

Figure 7 shows $L_{X}(2-10 \mathrm{keV})$ versus $v L_{\gamma}(12 \mu \mathrm{m})$ for our sample. We note that $v L_{v}(12 \mu \mathrm{m})$ is computed using the Spitzer spectra. S1s, S2s, and PGQSOs (panel a) follow the same relation as previously found for AGN (from Asmus et al. 2011). 


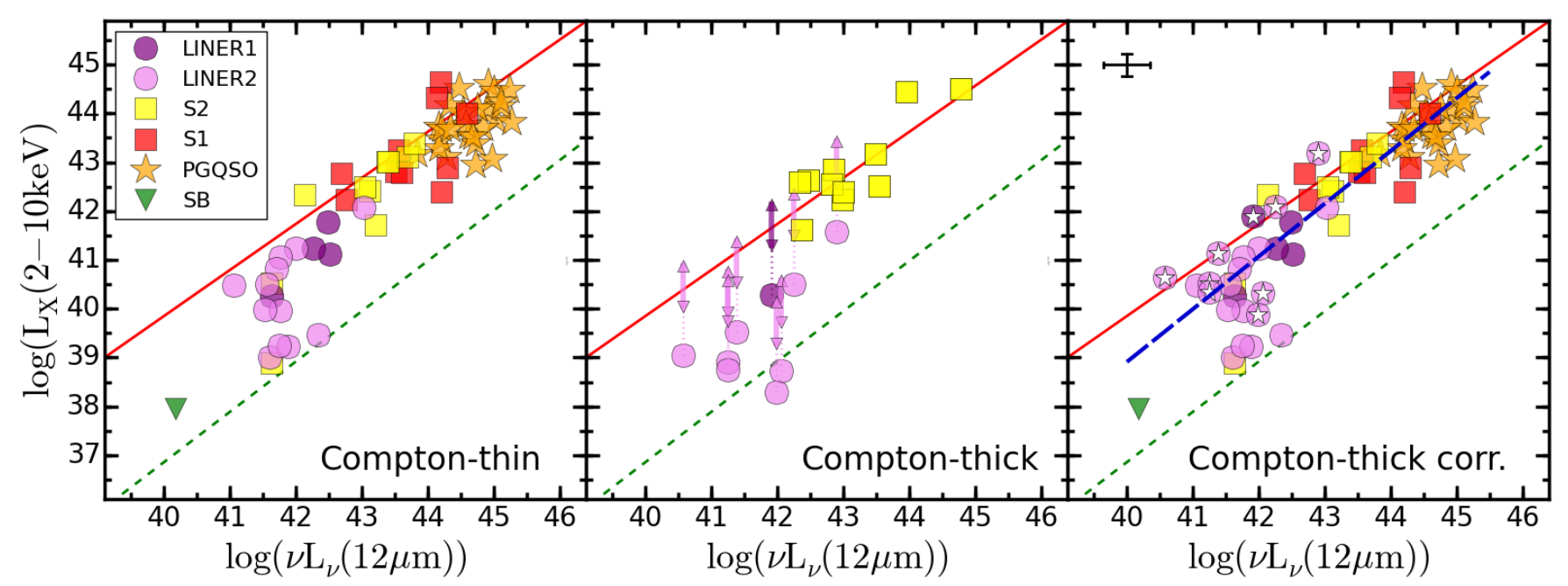

Fig. 8. 2-10 keV luminosity versus the $12 \mu \mathrm{m}$ luminosity, both on logarithmic scales for Compton-thin (left), Compton-thick (middle), and the full sample after Compton-thickness correction (right). The typical error for these measurements is shown as a cross in the top left corner of the right panel. The continuous-red and dashed-green lines show the best fit correlations for AGN and SBs, respectively, reported by Asmus et al. (2011). The long-dashed blue line shows the linear fit to the full sample. Grey arrows mark objects with reported upper limits on the X-ray luminosity. Small double arrows in the middle panel show the expected locus if the real intrinsic $2-10 \mathrm{keV}$ luminosity is $10-70$ times higher than estimated.

The Pearson correlation coefficient for them is $r=0.82$ $\left(P(\right.$ null $\left.)=4.7 \times 10^{-18}\right)$, and the best linear fit is very close to what is reported by Asmus et al. (2011) using high spatial resolution, mid-IR images. This correlation therefore holds for most S1s, S2s, and PG QSOs even with the relatively low spatial resolution of the Spitzer/IRS spectra. This may suggest that for these sources, the AGN continuum dominates the Spitzer/IRS emission. Only two S2s (NGC 3621 and NGC 4725) fall close to the correlation found for SB nuclei (also from Asmus et al. 2011). In our study NGC 3621 was classified as SB-dominated and NGC 4725 as AGN-dominated (see Sect. 4). SB nuclei (green upside down triangles in panel b in Fig. 7) also fall into the expected correlation for them with a correlation coefficient $r=0.83\left(P(\right.$ null $\left.)=4.5 \times 10^{-11}\right)$. None of them are compatible with the AGN correlation. Thus, this correlation seems to be very effective for distinguishing pure AGN emission from SB emission.

The bottom panels of Fig. 7 show $L_{X}(2-10 \mathrm{keV})$ versus $v L_{v}(12 \mu \mathrm{m})$ for LINERs. We split the plot into the AGNdominated (panel c) and the SB-dominated (panel d) LINERs. Most LINER nuclei are placed between the AGN and the SB linear relations. The linear correlation for them is not significant $\left(r=0.68\right.$ and $P($ null $\left.)=8 \times 10^{-6}\right)$. Low-luminosity LINERs are near SB correlation and high-luminosity LINERs are parallel to the AGN correlation. This could be due to an underestimation of the X-ray luminosity (see below).

Some LINERs behave as AGN, while some others are similar to SBs (see Sect. 4). Many of the LINER nuclei classified as SB-dominated are placed along the SB correlation (all the Type1 LINERs). Again, this confirms our method described in Sect. 4 as a good tool for distinguishing SB- from AGN-dominated spectra. However, three LINER2s (NGC 3079, NGC 6240, and NGC 7130) are placed in the AGN correlation but were classified as SB-dominated in the mid-IR. Interestingly, all of them are known Compton-thick AGNs. This suggests that the $12 \mu \mathrm{m}$ flux could be AGN-dominated although the full Spitzer/IRS spectrum is SB-dominated. Therefore, irrespective of the mid-IR spectral shape, the $12 \mu \mathrm{m}$ flux is a good tracer of the AGN power (e.g. González-Martín et al. 2013).
The inclusion of LINERs in this correlation allows it to be validated at lower luminosities $\left(L_{\mathrm{X}}(2-10 \mathrm{keV})<10^{42} \mathrm{erg} \mathrm{s}^{-1}\right.$, see also Mason et al. 2012). The final linear correlation for AGNdominated spectra is

$\log \left(L_{\mathrm{X}}\right)=(-12.34 \pm 0.05)+(1.26 \pm 0.01) \log \left(v L_{v}(12 \mu \mathrm{m})\right),(2)$

which is very significant $\left(r=0.92\right.$ and $P($ null $\left.)=2 \times 10^{-28}\right)$. However, the slope of this correlation is steeper $(\sim 1.26)$ than previously found $(\sim 1.06$, see Asmus et al. 2011). This excess in mid-IR luminosity for faint LINERs has already been found by Mason et al. (2012). They argue that this discrepancy could be due to optically thin material that obscures the inner parts of the AGN because most of them showed silicate features in emission. However, our faint LINERs do not show silicate features in emission on average (see Sect. 5 and Fig. 6).

The most natural explanation is that the Compton-thick nature of some low-luminosity AGNs results in an underestimation of the true $L_{X}(2-10 \mathrm{keV})$ of these sources. These $L_{X}(2-10 \mathrm{keV})$ estimates come from studies using the spectra of LINERs at energies below $10 \mathrm{keV}$. However, Compton-thick sources show the bulk of the AGN power at energies above $10 \mathrm{keV}$. The intrinsic luminosity could be 10-70 times higher than the estimated using only energies below $10 \mathrm{keV}$ in the Compton-thick scenario (Maiolino et al. 1998). González-Martín et al. (2009b) classified around $50 \%$ of their LINER sample as Compton-thick candidates. Figure 8 shows the AGN-dominated objects in our study attending to their Compton-thin (left) and Compton-thick (middle) classification. Most of the Compton-thin sources are close to the previously reported correlation for AGN. Comptonthick $\mathrm{S} 2 \mathrm{~s}$ are nicely placed along the AGN correlation found by Asmus et al. (2011). This is expected because the X-ray luminosities included for S2s are all corrected for their Comptonthick nature (most of them included in Goulding et al. 2012), either using X-ray measurements above $10 \mathrm{keV}$ or assuming a factor between the observed and intrinsic X-ray luminosity for other Compton-thick AGNs (see Panessa et al. 2006, for details in this conversion factor). Compton-thick LINER candidates tend to be shifted towards X-ray luminosities lower than predicted for the AGN correlation. However, most of them are not consistent with the SB correlation either. The double 
arrows of Fig. 8 (middle panel) show their expected locus if the X-ray luminosity were $\sim 10-70$ times higher. Most of the Compton-thick LINERs can be placed in the AGN correlation if the correction is applied. The linear fit to the entire sample, once the intrinsic X-ray luminosity is corrected, results in $L_{\mathrm{X}}(2-10 \mathrm{keV})$ (intrinsic) $=40 \times L_{\mathrm{X}}(2-10 \mathrm{keV})$ (observed) for Compton-thick LINERs (marked as white stars in Fig. 8, right panel), which is very close to the linear relation found for AGN. Nonetheless, four LINERs and one S2 still remain very close to the SB correlation.

\section{Discussion}

The nature of LINER nuclei has been extensively studied since they were firstly discovered by Heckman (1980). Using multiwavelength information and several techniques, such as compactness, hardness, and variability, we now know that a large number of them host an AGN (Maoz et al. 2005; GonzálezMartín et al. 2009a,b; Ho 2008). However, what makes them a unique class is still unknown. Singh et al. (2013) show that postmain sequence stars might be an important contributor to the optical frequencies. Interestingly, they argue that this population might be present in more powerful AGN, although it is outshone by the AGN itself. At X-rays, several authors have pointed to obscuration as one of the main ingredients for their different nature (Dudik et al. 2009; González-Martín et al. 2009b). Therefore, mid-IR frequencies are key to studying this obscuration since the emission absorbed at optical and UV frequencies is expected to be reprocessed at those wavelengths.

We have compiled a sample of 40 mid-IR spectra of LINERs observed with Spitzer/IRS and compared them to samples of SBs, S2s, S1s, and PG QSOs. Although the low spatial resolution of these Spitzer/IRS spectra is a disadvantage, we have been able to isolate SB-dominated from those that are not by using well known mid-IR tools (see Sect. 4). We focus this discussion on two main issues about the obscuration of LINERs in light of the present results: (1) Compton-thickness and (2) torus signatures in LINERs.

\subsection{Compton thickness}

González-Martín et al. (2009b) show that up to 50\% of their LINER sample might be Compton-thick, i.e. with such a high obscuration that the intrinsic continuum of the AGN is fully suppressed at energies below $10 \mathrm{keV}$. This was done using indirect arguments such as the [O III] $\lambda 5007 \AA$, the X-ray flux-ratio, or the EW of the neutral FeK $\alpha$ line at $6.4 \mathrm{keV}$. However, this is not yet confirmed with direct observations because LINERs are too faint to be observed above $10 \mathrm{keV}$ with the open X-ray instrumentation to the community ${ }^{11}$.

We have taken advantage of using the X-ray to mid-IR correlation found for AGN to study the Compton-thick nature of LINERs. LINERs classified as Compton-thick candidates by González-Martín et al. (2009b) are systematically located below the relation found for AGN (see Fig. 8, middle panel). This is naturally explained if they are indeed Compton-thick AGN, since their X-ray intrinsic luminosity is underestimated by a factor of 10 or more. This has already been found by Mason et al. (2012) for a few objects. Four additional LINERs and one Seyfert also have lower X-ray luminosities that expected according to their mid-IR luminosities (see Fig. 8, left panel). Thus,

\footnotetext{
${ }^{11}$ NuSTAR satellite is able to observe these faint source, but it is part of a close collaboration.
}

either they host a Compton-thick nucleus or are SB-dominated. This suggests that the actual fraction of Compton-thick LINERs may be even higher than previously inferred (González-Martín et al. 2009b). This large amount of obscuration might explain why post-main sequence stars dominate the optical spectrum in LINERs (Singh et al. 2013), while the AGN completely dominates the emission in powerful and/or less obscured AGN.

Observationally, a large number of AGNs in the local Universe are obscured by Compton-thick gas (Maiolino et al. 1998; Matt et al. 2000). From the theoretical point of view, a sizeable population of mildly Compton-thick sources is postulated in all the AGN synthesis models for the X-ray background in order to match the intensity peak of the XRB spectrum at about $30 \mathrm{keV}$ (Comastri 2004). These theoretical and observational evidence are consistent with the results of the present paper. Furthermore, the fraction of Compton-thick Seyferts is lower than reported in LINERs (and confirmed in this analysis). This is fully consistent with the paradigm in which the fraction of obscured sources increases when the luminosities decrease in the high-redshift Universe (Ueda et al. 2001) and in the local Universe (Shinozaki et al. 2006).

\subsection{Torus in LINERs}

We have found that the average mid-IR spectrum of LINERs is markedly different from those of SBs, S1s, S2s, or PGQSOs (see Fig. 5). The former shows a rather flat continuum from $6 \mu \mathrm{m}$ until $28 \mu \mathrm{m}$. On top of this flat continuum, prominent emission lines of [Ne II], [Ne III], [S III], and [O IV] are clearly seen. The [O IV] emission line suggests there is AGN emission, although it could be explained by another emission mechanism (see PereiraSantaella et al. 2010). The PAH features are also clearly seen. They indicate a non-negligible contribution of star formation to the mid-IR spectra of LINERs. However, note that star formation cannot be the dominant process for them because the SB mid-IR continuum is much steeper ${ }^{12}$ (see right panel of Fig. 5).

We have also found that the shape of the average mid-IR spectrum of LINERs does not depend on its Compton-thickness or AGN nature at X-rays (see Fig. 6). It depends on the optical type; i.e. the average mid-IR spectrum of LINER1s is different to that of LINER2s. However, the scatter around the mean in LINER1s is large, indicating that probably they are not a well-defined class. The best distinction between two subclasses is found for bright and faint LINERs, above and below $L_{\mathrm{X}}>1 \times 10^{41} \mathrm{erg} / \mathrm{s}$, respectively (Fig. 6).

The mid-IR spectrum of AGN is expected to be dominated by dust re-emission of optical/UV emission. In the unified model of AGNs, this dust is distributed in a dusty torus (Antonucci 1993). This torus was first postulated as having a smooth doughnut-like structure. However, nowadays the preferred scenario is a clumpy structure, i.e. one made of dusty clouds within a toroidal distribution (Krolik \& Begelman 1988; Pier \& Krolik 1992). Nenkova et al. (2002) developed a formalism for handling radiative transfer in clumpy media and applied it to the IR emission from the AGN dusty torus. Nenkova et al. (2008) applied this formalism to develop a set of models for this clumpy medium. They show that clumpy torus models are consistent with current AGN observations if they contain a number of dusty clouds along the equatorial axis of $N_{\mathrm{o}} \sim 5-15$, each with an optical depth of $\tau_{\mathrm{V}} \sim 30-100$. Ramos Almeida et al. (2011) modelled the IR SED for a sample of S1s and S2s by Nenkova et al. (2008) dusty torus models, using a Bayesian

${ }^{12}$ In this discussion SB-dominated LINERs are excluded, see Sect. 4. 


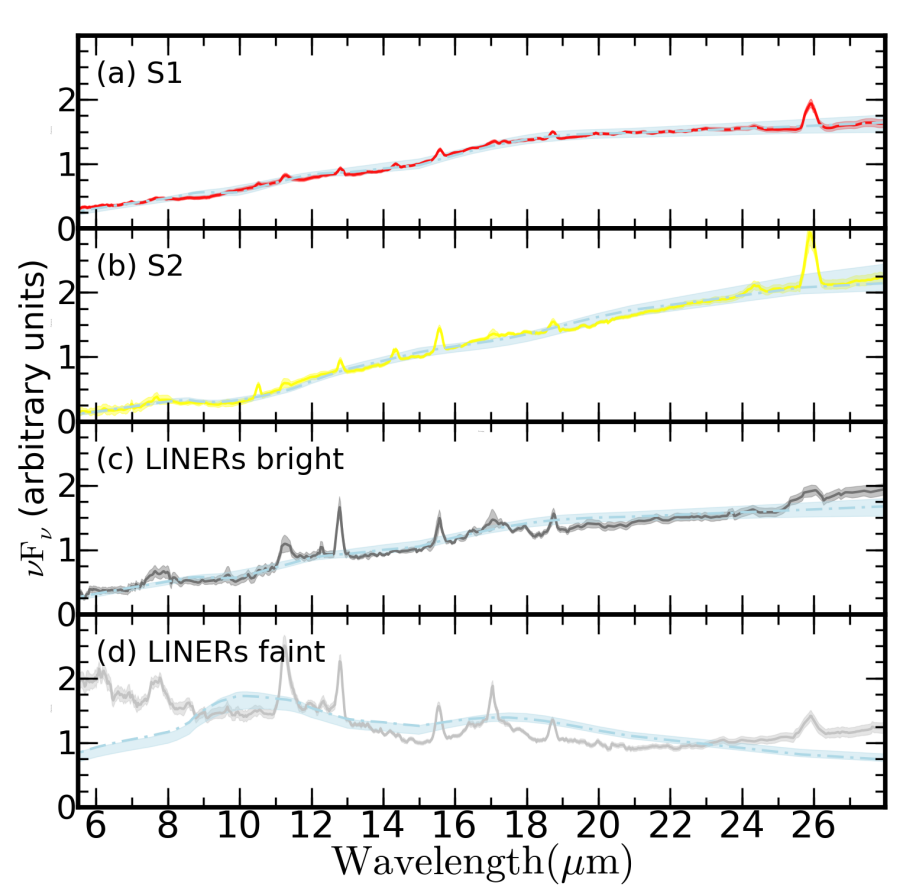

Fig. 9. Best fit to clumpy models (dot-dashed light-blue line) for the average spectra, from top to bottom, of S1s (panel a)), S2s (panel b)), bright LINERs (panel c)), and faint LINERs (panel d)). Bright LINERs are those with an X-ray luminosity above $L_{\mathrm{X}}>1 \times 10^{41} \mathrm{erg} / \mathrm{s}$. All the spectra are normalised to their flux at $15 \mu \mathrm{m}$. The shaded light-blue area shows the lower and upper bounds obtained with the clumpy models (see text).

inference tool called BayesClumpy (Asensio Ramos \& Ramos Almeida 2009). They find that the IR SED of both S1s and S2s are well fitted by these clumpy torus models, although their intrinsic properties are different (larger covering factor for S2s than for S1s, see also Alonso-Herrero et al. 2011).

We used the BayesClumpy tool to investigate whether clumpy models could fit our average mid-IR spectra. The median best-fit models found for the average mid-IR spectra are shown in Fig. 9, together with $68 \%$ confidence intervals for all the parameters (see Ramos Almeida et al. 2009, for more details on the modelling). As already shown by Ramos Almeida et al. (2011), the continuum shape of S1s and S2s are very represented well by clumpy models (panels a and b). Bright LINERs (i.e. $L_{\mathrm{X}}(2-10 \mathrm{keV})>1 \times 10^{41} \mathrm{erg} / \mathrm{s}$, panel c) can also be reproduced by these models. However, faint LINERs (panel d) are poorly represented by them. This may suggest that the optically thick torus emission may no longer dominate the mid-IR emission of faint LINERs.

In recent years, several pieces of observational evidence have supported the scenario in which a single continuum distribution of clouds within a wind is responsible for both the BLR and the dusty torus (Elitzur \& Shlosman 2006, and references therein). In this scenario, the difference between the BLR and dusty torus is just a change in the composition of this wind at the dust sublimation radius. An immediate consequence of the disk-wind scenario is the prediction that the torus and the BLR disappear at bolometric luminosities below $L_{\text {bol }} \sim 10^{42} \mathrm{erg} / \mathrm{s}$ (Elitzur \& Shlosman 2006). This limit is consistent with our results when assuming a conversion factor between the bolometric luminosity and X-ray luminosity of $L_{\mathrm{bol}} / L_{\mathrm{X}}(2-10 \mathrm{keV}) \sim 10$ (see Ho 2008).

\subsection{Alternative source of emission at mid-IR}

If faint LINERs are no longer dominated by the torus emission, what is the mechanism responsible for the mid-IR emission? This is a question for which we do not have a clear answer. Plausible contributors to the mid-IR emission are (1) jet emission in the form of synchrotron radiation, (2) host galaxy contributors; and (3) advection-dominated accretion flows (ADAFs). We have excluded the dust shell models because we do not see emission silicate features in the average mid-IR spectrum of faint LINERs, although it might be relevant for some of them (e.g. NGC 3998, Mason et al. 2013). Figure 10 compares the shape of the mid-IR spectrum of faint LINERs with several mechanisms discussed in this section. All of them are scaled to the emission at $15 \mu \mathrm{m}$.

\subsubsection{Host galaxy contribution}

We discard star formation as the dominant emission since the SB-dominated LINERs were not considered in the analysis, although it can still be present in a fraction, as suggested by the PAH emission. Alternatively, it could be linked to the diffuse emission coming from the galaxy. LINERs are generally found in elliptical galaxies (see Carrillo et al. 1999). Indeed in a very few cases, faint LINERs are hosted in late type galaxies (see Sect. 5). To compare the mid-IR spectra of LINERs with elliptical galaxies, we chose the Spitzer/IRS spectrum of the prototypical elliptical galaxy NGC $1407^{13}$. Although the general slope of faint LINERs resembles that of an elliptical galaxy, it lacks PAH features and emission lines seen in faint LINERs.

Singh et al. (2013) find that a large number of the optical spectra of many LINERs could come from post- asymptotic giant branch (post-AGB) stars. To investigate this deeply, Fig. 10 (bottom) compares the spectrum of faint LINERs and SBs with different stages of the AGB stars.

Our average LINER faint spectrum shows PAH features (7.7, 8.6, 11.3, and $17 \mu \mathrm{m}$ ), together with emission lines (e.g. [Ne II], [Ne III], [S III], and [O IV]). This is very different for AGB stars (see the spectrum of an AGB star SMP SMC 18 in Fig. 10, bottom), which show featureless continuum-dominated spectra (Yang et al. 2004; Sloan et al. 2006). Post-AGB stars (also called photo-planetary nebulae, proto-PNe) show some nebular emission lines and/or PAH features (see the spectrum of post-AGB star IRAS $01005+7910$ in Fig. 10, bottom). However, they show a peak on the continuum emission above $25 \mu \mathrm{m}$, and they lack the [O IV] line at $26 \mu \mathrm{m}$ seen in faint LINERs (Cerrigone et al. 2009).

The average spectrum of faint LINERs is more similar to evolved carbon-rich $\mathrm{PNe}$, where the continuum is less prominent, but PAH features and highly ionised nebular lines (e.g. [O IV]) are still present (Stanghellini et al. 2007). Figure 10 (bottom) shows the comparison between the average spectrum for faint LINERs and the prototype carbon-rich PNe SMP LMC 79. Although less prominent, the continuum of these carbon-rich PNe is still present. Stanghellini et al. (2012) showed that the dust continuum of carbon-rich PNe is well fitted with a blackbody model with a temperatures ranging $T \sim 100-160 \mathrm{~K}$. Moreover, at least $10^{6} \mathrm{PNe}$ are needed if they are the only ones responsible for the mid-IR luminosity of faint LINERs, since PNe typically show IR luminosities of $L_{\mathrm{IR}} \sim 1 \times 10^{34} \mathrm{erg} \mathrm{s}^{-1}$ (Stanghellini et al. 2012). Alternatively, a combination of

\footnotetext{
${ }^{13}$ We chose NGC 1407 because it is one of the few elliptical galaxies with full coverage of the mid-IR spectrum.
} 

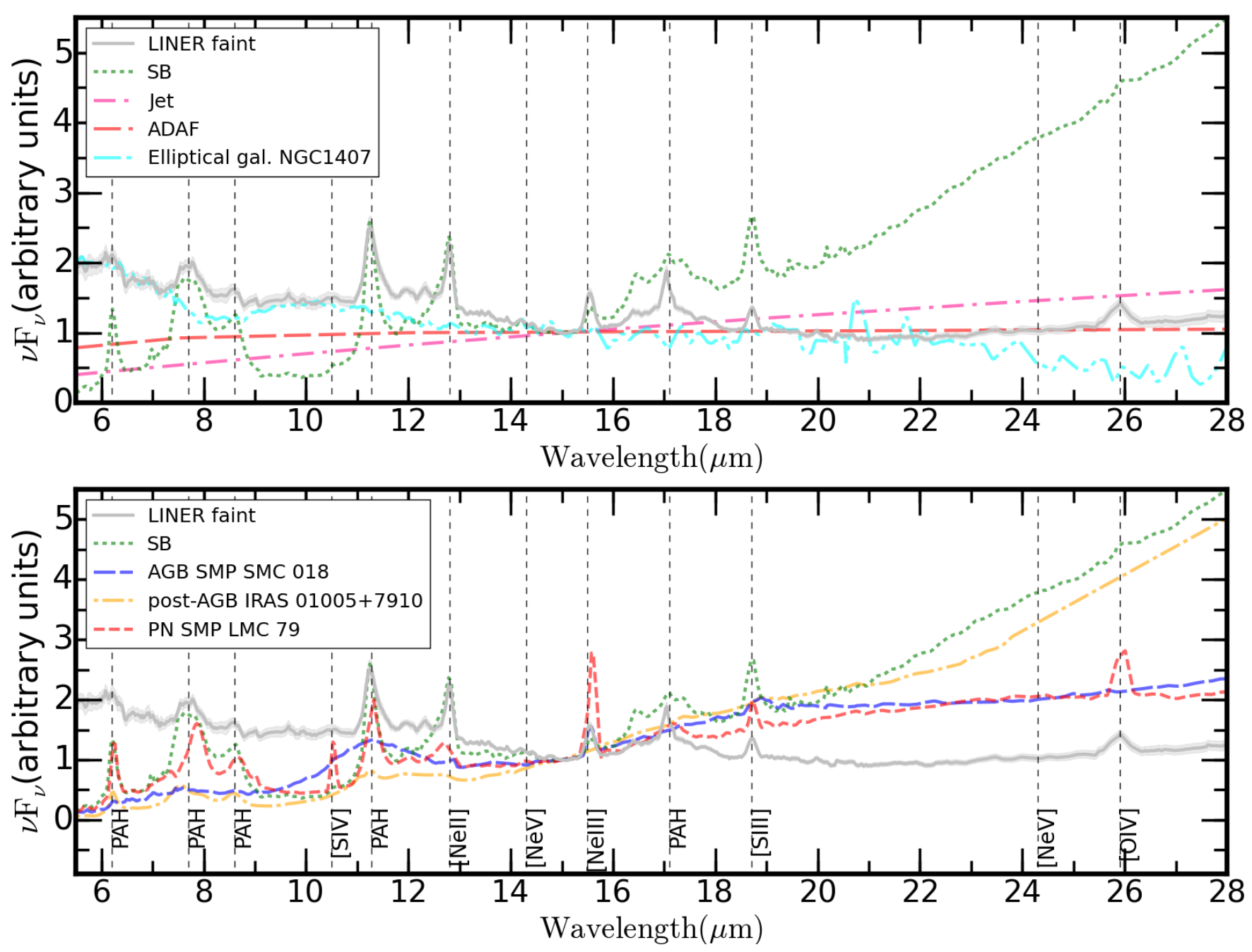

Fig. 10. Top: Mid-IR spectrum of faint LINERs (grey continuous line), SBs (green dotted line), elliptical galaxy NGC 1407 (cyan dot-dashed line), synchrotron emission of a jet (pink dot-dashed line), and ADAF (red dashed line). Bottom: Mid-IR spectrum of faint LINERs (grey continuous line), SBs (green dotted line), AGB star SMP SMC 018 (long-dashed blue line), post-AGB star IRAS 01005+7910 (dot-dashed orange line), and PN SMP LMC 79 (short-dashed red line).

elliptical galaxies (top panel of Fig. 10) and carbon-rich PNe could also reproduce the mid-IR average spectrum of faint LINERs. Finally, we checked the $[\mathrm{OI}] / \mathrm{H} \alpha$ ratio for our subsample of faint LINERs, and in eight out of the 22 of them the ratio is too high (i.e. $[\mathrm{OI}] / \mathrm{H} \alpha>0.25$ ) to be explained by post-AGB stars (Cid Fernandes et al. 2004). Thus, none of these stages of AGB stars can fully accomplish this for the observed features of faint LINERs, although a combination of several of them can explain the mid-IR spectrum for some of these faint LINERs.

Interestingly, a combination of carbon-rich post-AGB stars and PNe could explain the SB-dominated spectra. Moreover, the average spectrum of SBs SB-dominated S2s and SB-dominated LINERs show very similar spectra (see Fig. 5). Thus, in all these cases a combination of carbon-rich post-AGB and evolved PNe might be relevant at mid-IR. This agrees with the findings by Singh et al. (2013), where post-AGB stars dominate the optical spectrum when the AGN is faint.

\subsubsection{Advection-dominated accretion flows}

ADAF mechanisms have been largely claimed as the main one responsible for the SED of LINERs and LLAGN in general (e.g. Nemmen et al. 2014, and references therein). In these models the accretion disk is truncated in the inner parts and replaced by a hot, geometrically thick, optically thin accretion flow (Narayan et al. 1998). This flow is radiatively inefficient for accreting material to the inner parts. ADAF models are quite complex in showing many observational parameters. To compare our average mid-IR spectra with these models, we chose the ADAF best fit to NGC 1097 reported by Nemmen et al. (2014). ADAF model (see Fig. 10) fails to reproduce the slope of the spectrum and also lacks the PAH features and emission lines seen in faint LINERs.

\subsubsection{Jet emission}

LLAGN are generally radio loud according to their SED (Ho 2008). Several authors have pointed out that the full SED could be explained as emission from the jet (e.g. NGC 1052, Fernández-Ontiveros et al. 2012). Mason et al. (2013) show that jet emission is relevant for radio-loud LLAGN. To test this hypothesis, we adopted a simplistic jet model based on internal shocks adapted for AGN (see Nemmen et al. 2006, 2014, and references therein). The parameters of the model are those used in Nemmen et al. (2014), assuming a power-law index distribution 
with index $p=2$. We have assumed that the optically thin part of the jet is the one dominating the mid-IR emission. Jet emission (similarly to the ADAF models) shows a spectral index that is opposite to the one observed in faint LINERs (see top panel of Fig. 10). However, a combination of jet and other mechanisms could also explain the average spectrum of faint LINERs.

In summary, the shape of the mid-IR continuum of faint LINERs resembles that of elliptical galaxies, with a plausible contribution of carbon-rich PNe. However, it cannot be entirely described by any of the emission mechanisms explained above. An AGN component might also be present at some level to account for lines like [O IV], which are sometimes suggested as indicative of AGN activity (see Dudik et al. 2009). The emission mechanism producing the [O IV] line, however, has been proposed as being unrelated to the AGN (Pereira-Santaella et al. 2010). Indeed it is present in the spectrum of carbon-rich $\mathrm{PNe}$ (see the bottom panel of Fig. 10).

\section{Conclusions}

We have analysed a sample of mid-IR spectra of 40 LINERs observed with Spitzer/IRS. We compared the LINER sample with PG QSOs, Seyferts (S1 and S2), and starburst (SB). The main results of this paper are:

- We have developed a methodology to exclude SB-dominated mid-IR spectra based on the silicate optical depth $\tau_{9.7 \mu \mathrm{m}}$, the strength of the $6.2 \mu \mathrm{m}$ PAH feature, and the steepness of the mid-IR spectra. We found that 25 out of the 40 LINERs do not show any signatures of being SB-dominated. The fraction is similar to that obtained for Type-2 Seyferts.

- LINERs fall into the X-ray to mid-IR correlation for AGN only if the Compton-thick candidates proposed in X-rays show an X-ray luminosity around 70 times higher than computed in X-rays. This is expected if they are confirmed as Compton-thick AGN (see Sect. 6). Furthermore, four LINERs not previously classified as Compton-thick candidates are also consistent with being Compton-thick according to the X-ray to mid-IR relation.

- Bright LINERs (i.e. $\left.L_{X}(2-10 \mathrm{keV})>10^{41} \mathrm{erg} / \mathrm{s}\right)$ tend to have an average mid-IR spectrum similar to that of Type1 Seyferts. However, faint LINERs (i.e. $L_{\mathrm{X}}(2-10 \mathrm{keV})<$ $\left.10^{41} \mathrm{erg} / \mathrm{s}\right)$ show a flatter average mid-IR spectrum with prominent emission lines. We suggest that this favours the disappearance of the dusty torus in LINERs with bolometric luminosities below $L_{\text {bol }} \simeq 10^{42} \mathrm{erg} / \mathrm{s}$, as predicted theoretically. Therefore, LINERs with bolometric luminosities below $L_{\text {bol }} \simeq 10^{42} \mathrm{erg} / \mathrm{s}$ might no longer be dominated by the torus in the mid-IR. Their mid-IR continuum emission resembles that of an elliptical galaxy although an AGN or a jet component, together with some star-forming contribution (post-AGB stars and PNe) might also be present at some level.

A final caveat is that a large number of LINERs seem to be Compton-thick in X-rays (see previous subsection), although the torus emission have disappeared for them. How is that possible? A natural explanation is that the Compton-thick material seen in X-rays is not produced in the dusty torus. Dusty material absorbs continuum radiation both in the UV/optical and $\mathrm{X}$-rays, and therefore the dusty torus might also be responsible for part of the X-ray obscuration. But dust-free gas attenuates just the X-ray continuum, so clouds inside the dust sublimation radius will provide additional obscuration only in this band. Conclusive evidence of such absorption comes from the short timescales for transit of X-ray absorbing clouds across the LOS, which establish the existence of obscuring clouds inside the dust sublimation radius (e.g. NGC 1365, Risaliti et al. 2009). A natural explanation is that the Compton-thick clouds seen in X-rays in faint LINERs are produced in dust-free regions within the dust sublimation radius. Could LINERs be at a stage where the torus structure is already gone while the BLR is still present? This would naturally explain why the optical spectrum of LINERs needs a population of post-main sequence stars, blocking the source of ionisation that produces NLR lines (Singh et al. 2013). Alternatively, this Compton-thick gas might not be related to the AGN, but rather to the host galaxy as a result of galaxy interactions. In favour of that, many of the well known Compton-thick AGN are hosted in galaxy pairs or clusters (e.g. NGC 3690, Goulding et al. 2012; González-Martín et al. 2013), or even binary nuclei(e.g. NGC 6240, Komossa et al. 2003). A closer look at the environment of Compton-thick AGN needs to be undertaken so as to shed more light on this possibility.

Acknowledgements. We thank the referee for useful comments and suggestions. The authors acknowledge Dr. Rodrigo Nemmen for his help on the SED models and Dr. A. García-Hernández for his advice on the mid-IR emission of AGB stars and PNe. Partially based on observations made with the Gran Telescopio Canarias (GTC), installed at the Spanish Observatorio del Roque de los Muchachos of the Instituto de Astrofísica de Canarias on the island of La Palma.This research has been supported by the Spanish Ministry of Economy and Competitiveness (MINECO) under the grant (project refs. AYA2013-42227-P, AYA 2012-39168-C03-01, and AYA 2010-15169) and by La Junta de Andalucía (TIC 114). AAH acknowledges support from grant AYA2012-31447. D.D. acknowledges support from grant 107313 from PAPIIT, UNAM. C.R.A. is supported by a Marie Curie Intra European Fellowship within the 7th European Community Framework Programme (PIEF-GA-2012-327934).

\section{References}

Alonso-Herrero, A., Ramos Almeida, C., Mason, R., et al. 2011, ApJ, 736, 82 Alonso-Herrero, A., Pereira-Santaella, M., Rieke, G. H., et al. 2013, ApJ, 765, 78

Alonso-Herrero, A., Ramos Almeida, C., Esquej, P., et al. 2014, MNRAS, 443, 2766

Antonucci, R. 1993, ARA\&A, 31, 473

Asensio Ramos, A., \& Ramos Almeida, C. 2009, ApJ, 696, 2075

Asmus, D., Gandhi, P., Smette, A., Hönig, S. F., \& Duschl, W. J. 2011, A\&A, 536, A36

Asmus, D., Hönig, S. F., Gandhi, P., Smette, A., \& Duschl, W. J. 2014, MNRAS, 439, 1648

Bassani, L., Dadina, M., Maiolino, R., et al. 1999, ApJS, 121, 473

Baum, S. A., Gallimore, J. F., O’Dea, C. P., et al. 2010, ApJ, 710, 289

Bianchi, S., Maiolino, R., \& Risaliti, G. 2012, Adv. Astron., 2012

Brandl, B. R., Bernard-Salas, J., Spoon, H. W. W., et al. 2006, ApJ, 653, 1129

Cappi, M., Panessa, F., Bassani, L., et al. 2006, A\&A, 446, 459

Carrillo, R., Masegosa, J., Dultzin-Hacyan, D., \& Ordoñez, R. 1999, Rev. Mex. Astron. Astrofis., 35, 187

Cerrigone, L., Hora, J. L., Umana, G., \& Trigilio, C. 2009, ApJ, 703, 585

Cid Fernandes, R., González Delgado, R. M., Schmitt, H., et al. 2004, ApJ, 605, 105

Comastri, A. 2004, Supermassive Black Holes in the Distant Universe, 308, 245

Dale, D. A., Smith, J. D. T., Armus, L., et al. 2006, ApJ, 646, 161

Deo, R. P., Crenshaw, D. M., Kraemer, S. B., et al. 2007, ApJ, 671, 124

Diamond-Stanic, A. M., \& Rieke, G. H. 2012, ApJ, 746, 168

Dopita, M. A., \& Sutherland, R. S. 1995, ApJ, 455, 468

Dudik, R. P., Satyapal, S., Gliozzi, M., \& Sambruna, R. M. 2005, ApJ, 620, 113

Dudik, R. P., Satyapal, S., \& Marcu, D. 2009, ApJ, 691, 1501

Elitzur, M., \& Shlosman, I. 2006, ApJ, 648, L101

Esquej, P., Alonso-Herrero, A., González-Martín, O., et al. 2014, ApJ, 780, 86

Ferland, G. J., \& Netzer, H. 1983, ApJ, 264, 105

Fernández-Ontiveros, J. A., Prieto, M. A., Acosta-Pulido, J. A., \& Montes, M. 2012, J. Phys. Conf. Ser., 372, 012006

Gandhi, P., Horst, H., Smette, A., et al. 2009, A\&A, 502, 457

Genzel, R., Lutz, D., Sturm, E., et al. 1998, ApJ, 498, 579

González-Martín, O., Masegosa, J., Márquez, I., Guerrero, M. A., \& Dultzin-Hacyan, D. 2006, A\&A, 460, 45 
González-Martín, O., Masegosa, J., Márquez, I., Guainazzi, M., \& Jiménez-Bailón, E. 2009a, A\&A, 506, 1107

González-Martín, O., Masegosa, J., Márquez, I., \& Guainazzi, M. 2009b, ApJ, 704, 1570

González-Martín, O., Rodríguez-Espinosa, J. M., Díaz-Santos, T., et al. 2013, A\&A, 553, A35

González-Martín, O., Díaz-González, D. J., Acosta-Pulido, J. A., et al. 2014, A\&A, 567, A92

Goulding, A. D., Alexander, D. M., Bauer, F. E., et al. 2012, ApJ, 755, 5

Grier, C. J., Mathur, S., Ghosh, H., \& Ferrarese, L. 2011, ApJ, 731, 60

Heckman, T. M. 1980, A\&A, 87, 152

Hernán-Caballero, A., \& Hatziminaoglou, E. 2011, MNRAS, 414, 500

Hernández-García, L., González-Martín, O., Márquez, I., \& Masegosa, J. 2013, A\&A, 556, A47

Hernández-García, L., González-Martín, O., Masegosa, J., \& Márquez, I. 2014, A\&A, 569, A26

Ho, L. C. 2008, ARA\&A, 46, 475

Ho, L. C. 2009 , ApJ, 699, 626

Ho, L. C., Filippenko, A. V., Sargent, W. L. W., \& Peng, C. Y. 1997, ApJS, 112 , 391

Hönig, S. F., Kishimoto, M., Gandhi, P., et al. 2010, A\&A, 515, A23

Hönig, S. F., Leipski, C., Antonucci, R., \& Haas, M. 2011, ApJ, 736, 26

Horst, H., Duschl, W. J., Gandhi, P., \& Smette, A. 2009, A\&A, 495, 137

Imanishi, M., Dudley, C. C., Maiolino, R., et al. 2007, ApJS, 171, 72

Kennicutt, R. C., Jr., Armus, L., Bendo, G., et al. 2003, PASP, 115, 928

Komossa, S., Burwitz, V., Hasinger, G., et al. 2003, ApJ, 582, L15

Krabbe, A., Böker, T., \& Maiolino, R. 2001, ApJ, 557, 626

Krolik, J. H., \& Begelman, M. C. 1988, ApJ, 329, 702

LaMassa, S. M., Heckman, T. M., Ptak, A., et al. 2012, ApJ, 758, 1

Lebouteiller, V., Barry, D. J., Spoon, H. W. W., et al. 2011, ApJS, 196, 8

Levenson, N. A., Sirocky, M. M., Hao, L., et al. 2007, ApJ, 654, L45

Levenson, N. A., Radomski, J. T., Packham, C., et al. 2009, ApJ, 703, 390

Lutz, D., Spoon, H. W. W., Rigopoulou, D., Moorwood, A. F. M., \& Genzel, R. 1998, ApJ, 505, L103

Maiolino, R., Salvati, M., Bassani, L., et al. 1998, A\&A, 338, 781

Maoz, D., Nagar, N. M., Falcke, H., \& Wilson, A. S. 2005, ApJ, 625, 699

Marinucci, A., Bianchi, S., Nicastro, F., Matt, G., \& Goulding, A. D. 2012, ApJ, 748,130

Masegosa, J., Márquez, I., González-Martín, O., et al. 2013, Rev. Mex. Astron. Astrofis. Conf. Ser., 42, 51

Mason, R. E., Lopez-Rodriguez, E., Packham, C., et al. 2012, AJ, 144, 11

Mason, R. E., Ramos Almeida, C., Levenson, N. A., Nemmen, R., \& Alonso-Herrero, A. 2013, ApJ, 777, 164

Matt, G., Fabian, A. C., Guainazzi, M., et al. 2000, MNRAS, 318, 173

Mendoza-Castrejón, S., Dultzin, D., Krongold, Y., González, J. J., \& Elitzur, M. 2015, MNRAS, 447, 2437

Müller-Sánchez, F., Prieto, M. A., Mezcua, M., et al. 2013, ApJ, 763, LL1
Narayan, R., Mahadevan, R., Grindlay, J. E., Popham, R. G., \& Gammie, C. 1998, ApJ, 492, 554

Nardini, E., Risaliti, G., Salvati, M., et al. 2008, MNRAS, 385, L130

Nemmen, R. S., Storchi-Bergmann, T., Yuan, F., et al. 2006, ApJ, 643, 652

Nemmen, R. S., Storchi-Bergmann, T., \& Eracleous, M. 2014, MNRAS, 438, 2804

Nenkova, M., Ivezić, Ž., \& Elitzur, M. 2002, ApJ, 570, L9

Nenkova, M., Sirocky, M. M., Ivezić, Ž., \& Elitzur, M. 2008, ApJ, 685, 147

Panessa, F., Bassani, L., Cappi, M., et al. 2006, A\&A, 455, 173

Peeters, E., Mattioda, A. L., Hudgins, D. M., \& Allamandola, L. J. 2004, ApJ, 617, L65

Pereira-Santaella, M., Diamond-Stanic, A. M., Alonso-Herrero, A., \& Rieke, G. H. 2010, ApJ, 725, 2270

Pier, E. A., \& Krolik, J. H. 1992, ApJ, 401, 99

Ramos Almeida, C., Levenson, N. A., Rodríguez Espinosa, J. M., et al. 2009, ApJ, 702, 1127

Ramos Almeida, C., Levenson, N. A., Alonso-Herrero, A., et al. 2011, ApJ, 731, 92

Ramos Almeida, C., Alonso-Herrero, A., Levenson, N. A., et al. 2014, MNRAS, 439, 3847

Ranalli, P., Comastri, A., \& Setti, G. 2003, A\&A, 399, 39

Risaliti, G., Salvati, M., Elvis, M., et al. 2009, MNRAS, 393, L1

Satyapal, S., Dudik, R. P., O’Halloran, B., \& Gliozzi, M. 2005, ApJ, 633, 86

Shi, Y., Rieke, G. H., Hines, D. C., et al. 2006, ApJ, 653, 127

Shinozaki, K., Miyaji, T., Ishisaki, Y., Ueda, Y., \& Ogasaka, Y. 2006, AJ, 131, 2843

Singh, R., van de Ven, G., Jahnke, K., et al. 2013, A\&A, 558, A43

Sloan, G. C., Kraemer, K. E., Matsuura, M., et al. 2006, ApJ, 645, 1118

Smith, J. D. T., Draine, B. T., Dale, D. A., et al. 2007, ApJ, 656, 770

Spoon, H. W. W., Marshall, J. A., Houck, J. R., et al. 2007, ApJ, 654, L49

Stanghellini, L., García-Lario, P., García-Hernández, D. A., et al. 2007, ApJ, 671,1669

Stanghellini, L., García-Hernández, D. A., García-Lario, P., et al. 2012, ApJ, 753,172

Stasińska, G., Vale Asari, N., Cid Fernandes, R., et al. 2008, MNRAS, 391, L29

Sturm, E., Rupke, D., Contursi, A., et al. 2006, ApJ, 653, L13

Ueda, Y., Ishisaki, Y., Takahashi, T., Makishima, K., \& Ohashi, T. 2001, ApJS, 133,1

Urry, C. M., \& Padovani, P. 1995, PASP, 107, 803

Veilleux, S., \& Osterbrock, D. E. 1987, ApJS, 63, 295

Veilleux, S., Rupke, D. S. N., Kim, D.-C., et al. 2009, ApJS, 182, 628

Véron-Cetty, M.-P., \& Véron, P. 2006, A\&A, 455, 773

Weedman, D. W., Hao, L., Higdon, S. J. U., et al. 2005, ApJ, 633, 706

Wu, Y., Charmandaris, V., Huang, J., Spinoglio, L., \& Tommasin, S. 2009, ApJ, 701,658

Yang, X., Chen, P., \& He, J. 2004, A\&A, 414, 1049

Younes, G., Porquet, D., Sabra, B., \& Reeves, J. N. 2011, A\&A, 530, A149

Pages 17 to 19 are available in the electronic edition of the journal at http://www . aanda. org 


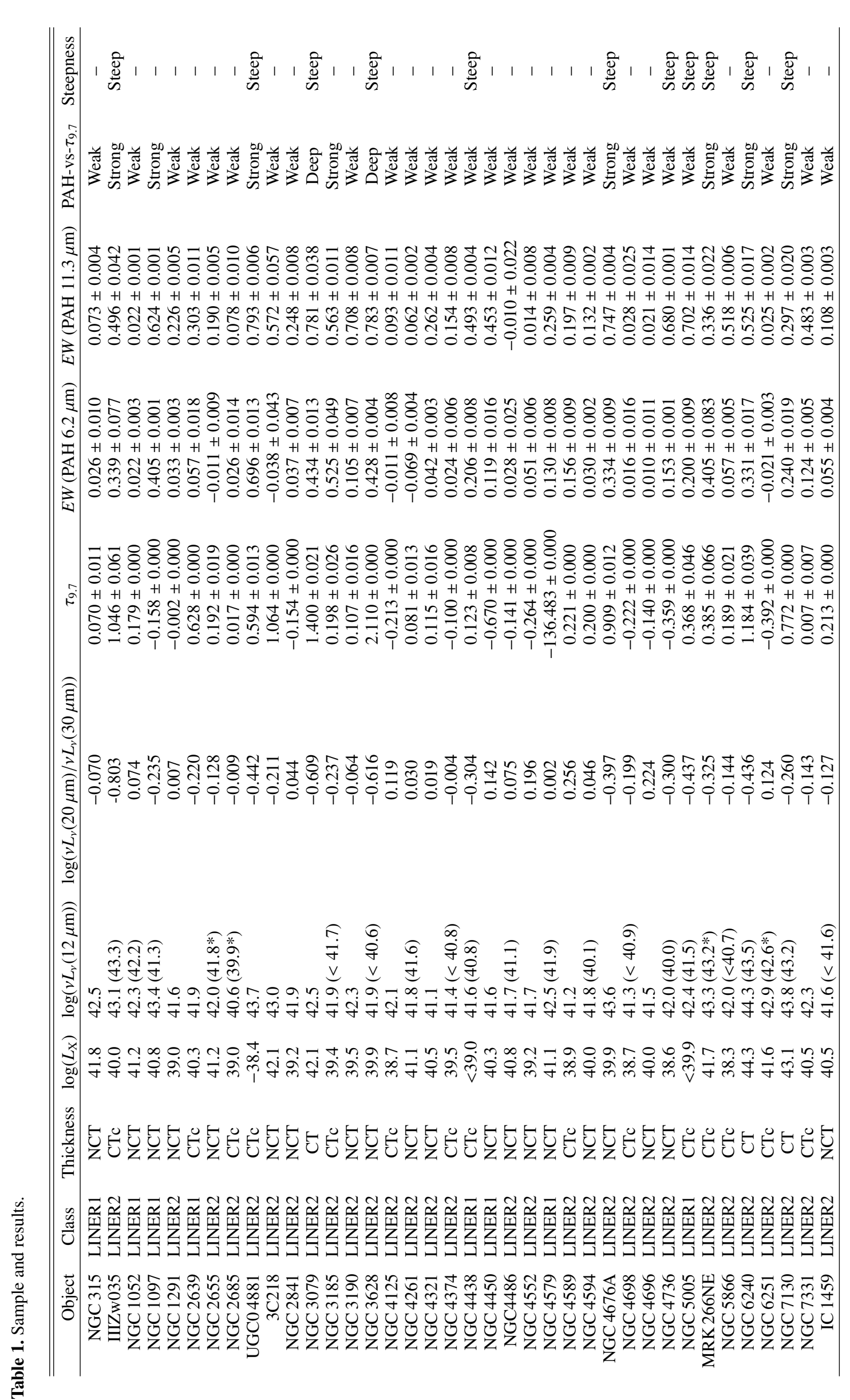

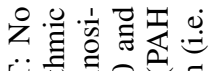

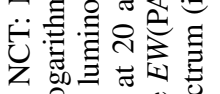

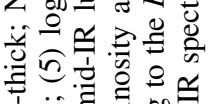

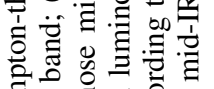

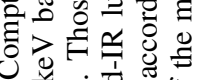
붕응

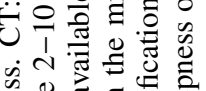

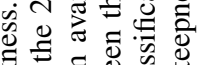

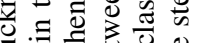

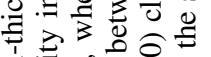
政

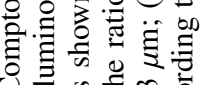

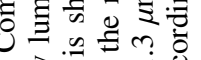

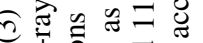

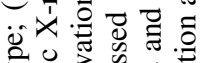
2.0 ‥ 용 0 o

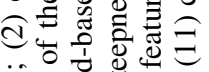

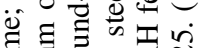

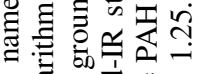
突品 혀웡 金焉

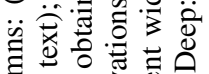

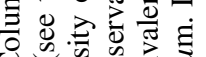
웅

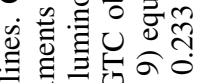

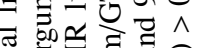

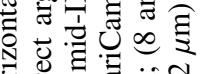

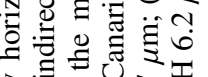
อ.

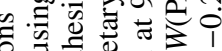

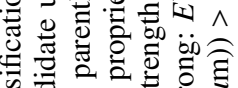

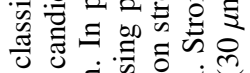

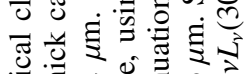

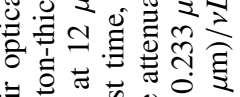

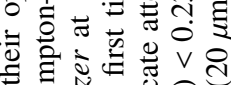

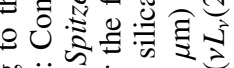
号

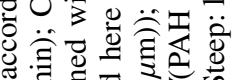

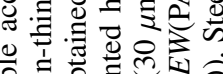

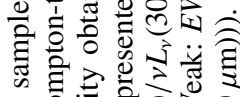
ठ ठ

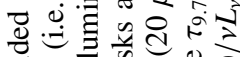

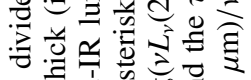

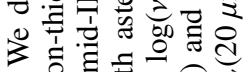

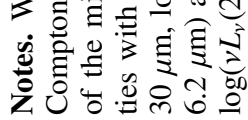




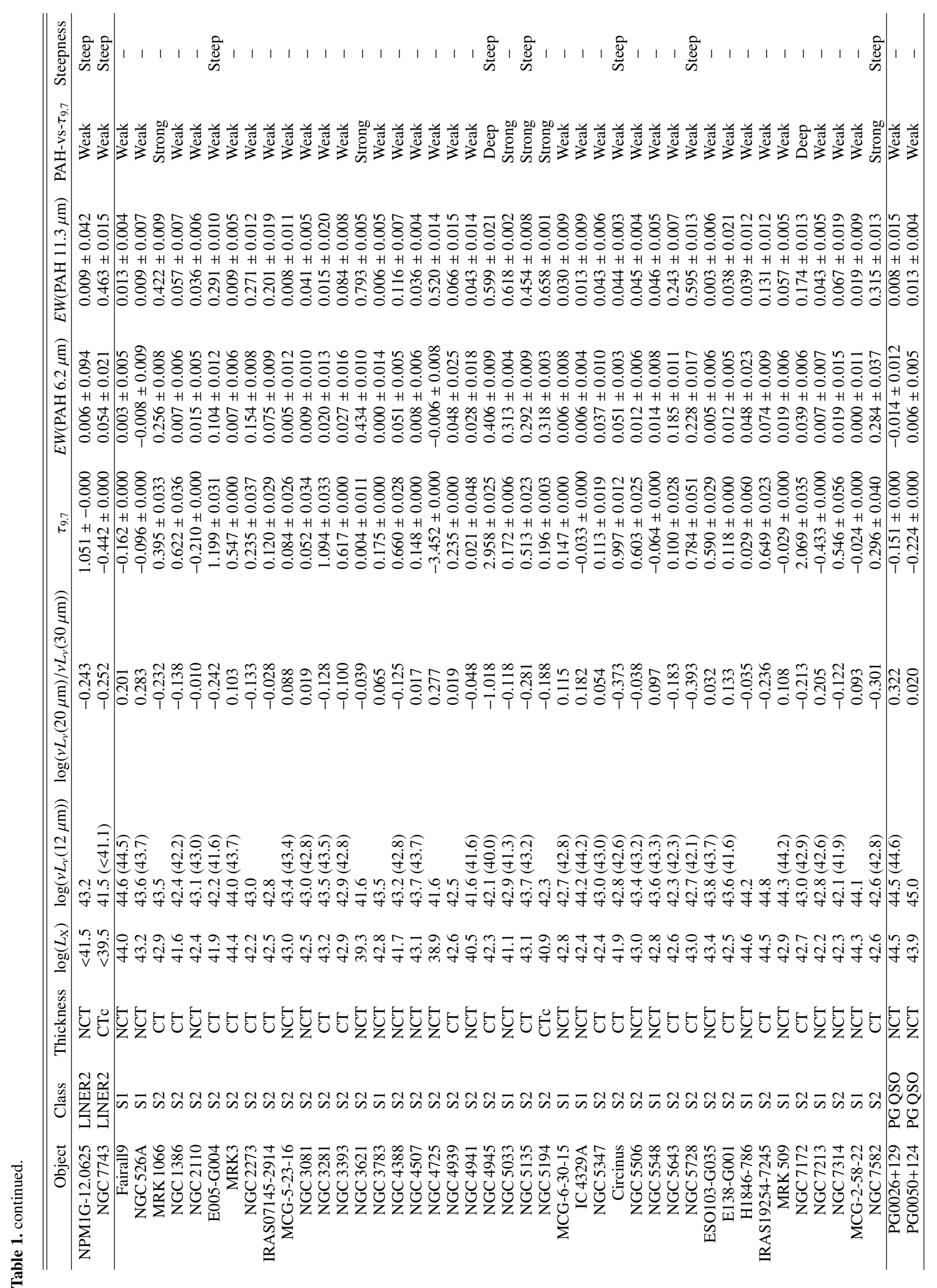




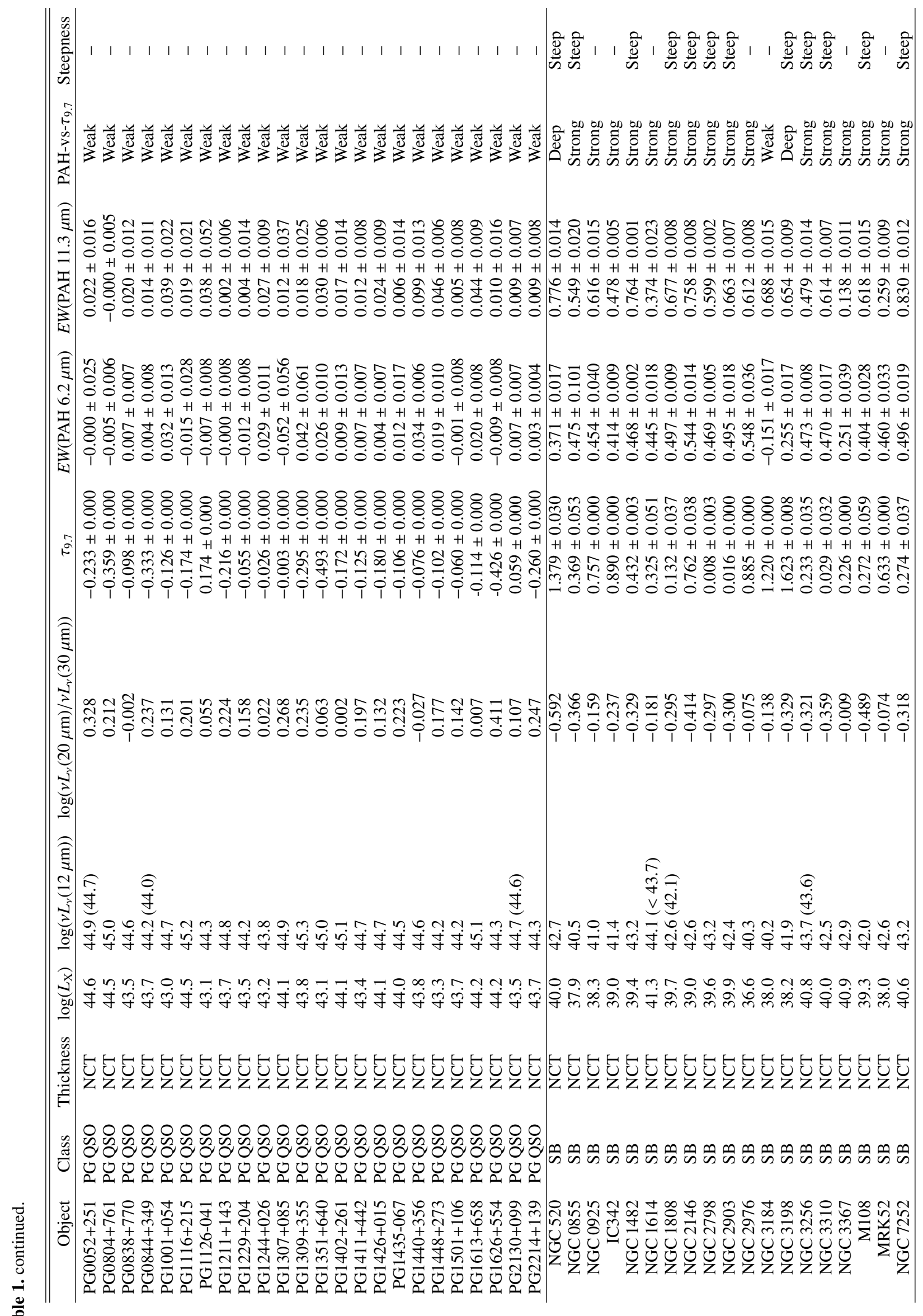

